\title{
Influence of Dietary Berberine on Liver Immune Response and Intestinal Health of Black Sea Bream (Acanthopagrus schlegelii) Fed with Normal and High-Lipid Diets
}

\author{
Lei Wang $\mathbb{D},{ }^{1,2}$ Gladstone Sagada $\mathbb{D}^{1},{ }^{1}$ Bingying Xu, ${ }^{1}$ Jinzhi Zhang, ${ }^{1}$ and Qingjun Shao $\mathbb{D}{ }^{1,3}$ \\ ${ }^{1}$ College of Animal Sciences, Zhejiang University, Hangzhou 310058, China \\ ${ }^{2}$ School of Ecology and Environment, Anhui Normal University, Wuhu 241000, China \\ ${ }^{3}$ Ocean Academy, Zhejiang University, Zhoushan 316021, China
}

Correspondence should be addressed to Lei Wang; leiwang@ahnu.edu.cn and Qingjun Shao; qjshao@zju.edu.cn

Received 25 October 2021; Revised 27 December 2021; Accepted 6 January 2022; Published 30 January 2022

Academic Editor: Erchao Li

Copyright (c) 2022 Lei Wang et al. This is an open access article distributed under the Creative Commons Attribution License, which permits unrestricted use, distribution, and reproduction in any medium, provided the original work is properly cited.

\begin{abstract}
This study designed the normal diet (Con), high-lipid diet (HL), and $50 \mathrm{mg} / \mathrm{kg}$ berberine supplemented Con and HL diets (named as ConB and HLB, respectively). Fingerling black sea bream (Acanthopagrus schlegelii) (initial weight $1.47 \pm 0.03 \mathrm{~g}$ ) were fed these four diets for 8 weeks, and the liver immune response and intestinal health were evaluated. Fish fed the HL diet had the lowest serum glutathione peroxidase and catalase activities, while berberine supplementation significantly improved serum catalase activity $(P<0.05)$. The expressions of tumor necrosis factor-alpha $(\operatorname{tnf} \alpha)$, nuclear factor-kappa $\mathrm{b}(n f-\kappa b)$, and interleukin 1 beta $(i l-1 \beta)$ were significantly increased by the HL diet, whereas berberine supplementation significantly downregulated these proinflammation cytokine expressions $(P<0.05)$. Berberine supplementation in normal and high-lipid diets significantly promoted hepatic anti-inflammation cytokines interleukin-10 (il-10) and transforming growth factor beta-1 (tgf $\beta$ - 1 ) expressions $(P<0.05)$. The intestinal expressions of $t n f \alpha, n f-\kappa b$, and $\operatorname{tg} f \beta-1$ were not significantly influenced by different treatments, while $\mathrm{Cu} / \mathrm{Zn}$-superoxide dismutase $(\mathrm{Cu} / \mathrm{Zn}$-sod) expression was significantly upregulated in the ConB group compared with the Con group. High-lipid diet reduced intestinal villus length. the thickness of muscularis mucosa, mucosal folds, and diversity and richness of intestinal microbiota but increased numbers of goblet cells and width of lamina propria. Berberine supplementation in high-lipid diet alleviated these pathological changes and rebalanced intestinal microbiota composition. At the genus level, fish fed the HL diet had significantly lower abundances of some probiotics such as Lactobacillus and Hydrogenophaga than that of the HLB group $(P<0.05)$. In conclusion, berberine supplementation in highlipid diet reduced liver inflammation, improved the antioxidative status of fish, and alleviated intestinal damage and intestinal microbiota alteration of black sea bream.
\end{abstract}

\section{Introduction}

Berberine is an isoquinoline alkaloid that can be extracted from Coptis chinensis, Berberis thunbergii, and Hydrastis Canadensis, and it exerts various pharmacological functions, such as antitumor, antifungal, cardioprotective, and antiinflammatory effects [1]. After oral administration, berberine enters the blood after structural transformation by intestinal microbiota. However, more than $90 \%$ of berberine remains in the intestine [2], where may be the main action site of berberine [3]. In traditional Chinese medicine, ber- berine is used for intestinal infections, especially bacterial diarrhea, mainly due to its antisecretory and antimicrobial characters [4]. In addition, berberine has a powerful free radical scavenging effect, and it can reduce the production of reactive oxygen species (ROS) via multiple ways, such as inhibiting ROS-producing oxidase enzymes, modulating the activities of several endogenous antioxidant enzymes, and quenching nitric oxide (NO), superoxide anion $\left(\mathrm{O}^{2-}\right)$, and the precariously reactively molecules peroxynitrite $\left(\mathrm{OONO}^{-}\right)$directly [5-7]. Moreover, berberine can directly downregulate the expressions of proinflammatory cytokines, 
such as interleukin-6 (IL-6), tumor necrosis factor- $\alpha$ (TNF$\alpha$ ), and IL- $1 \beta$, as well as indirectly affect the expressions of activator protein 1 (AP-1), nuclear factor-kappa B (NF- $\kappa \mathrm{B})$, the AMP-activated protein kinase (AMPK) signaling pathways, and the mitogen-activated protein kinase- (MAPK-) mediated pathways to decrease the inflammation responses $[4,8]$. Thus, berberine is an effective antioxidant and antiinflammation drug; it has the potential to be a functional feed additive for improving fish antioxidative and immune status.

The gut microbiota is a symbol representing health status ([9]); it can improve energy expenditure, nutrient absorption, and immune response, as well as inhibit proliferation of pathogens and keep the integrity of intestinal epithelium, which are beneficial for the host $[10,11]$. It has been proved that berberine altered gut microbiota to influence the metabolic phenotypes in rats $[2,12,13]$. In mice, berberine exerts its hepatic lipid-lowering effect partly through regulating microbial bile acid metabolism and the microbiota-gut-brain axis [14]. And berberine administration could alter intestinal and fecal Bacteroidetes and Firmicutes richness in mice, which are related to intestinal energy harvest $[15,16]$. Also, a study in grass carp (Ctenopharyngodon idella) revealed that administration of berberine reduced serum glucose, total cholesterol, and triglyceride contents by modulating the gut microbiota [3]. Yu et al. [17] observed that berberine improved the intestinal barrier stressed by a high-carbohydrate or high-fat diet by regulating the intestinal microbiota in blunt snout bream (Megalobrama amblycephala). Therefore, berberine exerts its multiple functions by modulating intestinal microbiota, and intestinal microbiota may be a target for berberine to maintain liver and intestine health threatened by high-lipid diet.

Black sea bream (Acanthopagrus schlegelii) is an important aquaculture species with many promising characteristics. Compared with carbohydrate, lipids are more important for black sea bream as an energy source, due to its high energy density and digestibility, and carnivorous fish has a limited ability in using dietary carbohydrate $[18,19]$. For fish, the foregut is the main site for lipid digestion and uptake [20], making it become an important target for studying lipid utilization. Previously, Wang et al. [21] reported that suitable dietary lipid levels could spare protein utilization, thus reducing the dietary protein requirement. However, excessive dietary lipid causes growth retardation and massive lipid accumulation in the liver, impairs immune and antioxidative capacity, disrupts the intestinal structural integrity $[17,22]$, and alters gut microbiota diversity [10, 23]. Previous studies have observed that high-lipid dietinduced intestinal and/or liver inflammation altered intestinal microbiota composition in different aquaculture species [24]. Berberine is an effective feed additive to treat fatty liver caused by high-lipid diet in blunt snout bream $[22,25,26]$ and zebrafish [27]. Previously, Wang et al. [19] reported that supplementation of $50 \mathrm{mg} / \mathrm{kg}$ berberine in high-lipid diet could effectively reduce hepatic lipid accumulation in black sea bream by upregulating lipolysis genes and downregulating lipogenesis gene expression. But berberine supplementa- tion did not influence growth performance and feed utilization in the abovementioned studies. However, those studies seldom focused on the alteration of the gut microbiota influenced by berberine in fish. Thus, we assumed that berberine may have a beneficial effect in maintaining the intestinal barrier by modulating the intestinal microbiota stressed by high-lipid diet.

Therefore, the purpose of this experiment was to explore whether berberine can relieve fish intestinal and liver damage, as well as antioxidant and immune status caused by high-lipid diet and whether the change of intestinal microbiota is involved in its alleviation of high lipid stress.

\section{Materials and Methods}

2.1. Experimental Diets and Feeding Trial. Four isonitrogenic diets ( $42 \%$ crude protein) were designed, and the formulation and proximate compositions are shown in Table 1. The control diet (Con) contained 11.1\% crude lipid, and the high-lipid diet (HL) had $20.3 \%$ crude lipid. $50 \mathrm{mg} / \mathrm{kg}$ berberine $\left(\mathrm{C}_{20} \mathrm{H}_{18} \mathrm{ClNO}_{4}, \mathrm{HPLC} \geq 98 \%\right)$, which was recommended as the suitable dosage in fish feed reported by Zhou et al. [26], was purchased from the Spring and Autumn Biotechnology Company (Nanjing, China) and added to Con and HL diets and named ConB and HLB, respectively. The main protein and lipid ingredients were purchased from the Minghui Feed Company (Hangzhou, China). All the ingredients were thoroughly grounded and sieved through a $178 \mu \mathrm{m}$ net. Then, the ingredients were mixed by a mixer, and water was added to increase viscidity. The diets were extruded through a $0.25 \mathrm{~mm}$ mold by a feed maker (Modle HKJ-218, HUARUI, Wuxi, China). All the diets were subsequently cooked by stream for ten minutes, then put on a clean floor, and dried by air conditioner and dehumidifier. Representative feed was collected for proximate composition analysis, and the remaining feed was sealed in plastic bags and put in $-20^{\circ} \mathrm{C}$ until use.

Black sea bream fingerlings were obtained from a farm in Zhou Shan, China. After being acclimated for 14 days, 420 healthy fish (initial weight $1.47 \pm 0.03 \mathrm{~g}$ ) were distributed into 12 tanks ( $400 \mathrm{~L}$ blue plastic tank, 35 fish per tank) with three replicates for each treatment. Seawater was obtained from the nearshore of Xixuan island (Marine Fisheries Research Institute of Zhejiang, Zhoushan, China). After sedimentation and filtration, water flowed into each tank at about $2 \mathrm{~L} /$ minute. The water condition was monitored every day and kept as temperature $27 \pm 1^{\circ} \mathrm{C}, \mathrm{pH} 8.2 \pm 0.1$, salinity $28 \pm 2$, and dissolved oxygen $>5 \mathrm{mg} / \mathrm{L}$. Then, the fish were fed experimental diets three times a day $(7: 00,12: 00$, and 16:00) for 8 weeks.

2.2. Sample Collection. At $24 \mathrm{~h}$ after the last feeding, all fish were anesthetized by MS-222 $(60 \mathrm{mg} / \mathrm{L})$, the foregut and liver samples were randomly collected from 12 fish of each tank. The blood sample was obtained from the caudal vein, and pooled blood samples were centrifuged at 10,000 $\mathrm{g}$ after a $2 \mathrm{~h}$ settlement at $4^{\circ} \mathrm{C}$. Three intestine and liver samples were put into $4 \%$ paraformaldehyde and $0.5 \%$ glutaraldehyde solution for histological observation. The other 
TABLE 1: Formulation and proximate compositions of experimental diets $^{1}$.

\begin{tabular}{|c|c|c|c|c|}
\hline Ingredients (g/kg) & Con & ConB & $\mathrm{HL}$ & HLB \\
\hline Fishmeal & 250.0 & 250.0 & 250.0 & 250.0 \\
\hline Wheat gluten meal & 50.0 & 50.0 & 50.0 & 50.0 \\
\hline Soy protein concentrate & 80.0 & 80.0 & 80.0 & 80.0 \\
\hline Fermented soybean meal & 150.0 & 150.0 & 150.0 & 150.0 \\
\hline Corn oil & 20.0 & 20.0 & 100.0 & 100.0 \\
\hline Fish oil & 50.0 & 50.0 & 50.0 & 50.0 \\
\hline Wheat flour & 150.0 & 150.0 & 150.0 & 150.0 \\
\hline Alpha starch & 40.0 & 40.0 & 40.0 & 40.0 \\
\hline Soy lecthin & 20.0 & 20.0 & 20.0 & 20.0 \\
\hline $\mathrm{Ca}\left(\mathrm{H}_{2} \mathrm{PO}_{4}\right)_{2} \cdot \mathrm{H}_{2} \mathrm{O}$ & 25.0 & 25.0 & 25.0 & 25.0 \\
\hline Alpha cellulose & 98.5 & 98.45 & 18.5 & 18.45 \\
\hline Vitamin premix ${ }^{2}$ & 7.5 & 7.5 & 7.5 & 7.5 \\
\hline Mineral premix ${ }^{3}$ & 7.5 & 7.5 & 7.5 & 7.5 \\
\hline $\mathrm{Y}_{2} \mathrm{O}_{3}$ & 1.0 & 1.0 & 1.0 & 1.0 \\
\hline Berberine & 0.0 & 0.05 & 0.0 & 0.05 \\
\hline Lysine & 4.0 & 4.0 & 4.0 & 4.0 \\
\hline Arginine & 6.0 & 6.0 & 6.0 & 6.0 \\
\hline Methionine & 8.0 & 8.0 & 8.0 & 8.0 \\
\hline Phytase & 0.50 & 0.50 & 0.50 & 0.50 \\
\hline Carrageenan & 2.0 & 2.0 & 2.0 & 2.0 \\
\hline Carboxymethyl cellulose & 30.0 & 30.0 & 30.0 & 30.0 \\
\hline Total & 1000.0 & 1000.0 & 1000.0 & 1000.0 \\
\hline \multicolumn{5}{|c|}{ Analyzed proximate composition (\% dry weight) } \\
\hline Crude protein & 42.51 & 41.71 & 42.40 & 41.95 \\
\hline Crude lipid & 11.19 & 11.13 & 20.26 & 20.37 \\
\hline Ash & 8.77 & 8.56 & 8.71 & 8.78 \\
\hline
\end{tabular}

${ }^{1}$ Con: control diet; ConB: control diet with berberine supplementation; HL: high-lipid diet; HLB: high-lipid diet with berberine supplementation. ${ }^{2}$ Vitamin premix (mg/kg): $\alpha$-tocopherol, 80 ; retinyl acetate, 40; cholecalciferol, 0.1; menadione, 15; niacin, 165; riboflavin, 22; pyridoxine $\mathrm{HC1}$, 40; thiamin mononitrate, 45; D-Ca pantothenate, 102, folic acid, 10; vitamin B-12, 0.9; inositol, 450; ascorbic acid, 150; $\mathrm{Na}$ menadione bisulphate, 15; thiamin, 5; choline chloride, 320 and p-aminobenzoic acid, 50. ${ }^{3}$ Mineral premix (mg/kg): $\mathrm{Na}_{2} \mathrm{SiO}_{3}, 0.4 ; \mathrm{CaCO}_{3}, 544.9 ; \mathrm{NaH}_{2} \mathrm{PO}_{4} \cdot \mathrm{H}_{2} \mathrm{O}$, 200; $\mathrm{KH}_{2} \mathrm{PO}_{4}, 200 ; \mathrm{MgSO}_{4} \cdot 7 \mathrm{H}_{2} \mathrm{O}, 10 ; \mathrm{MnSO}_{4} \cdot \mathrm{H}_{2} \mathrm{O}, 4 ; \mathrm{CuCl}_{2} \cdot 2 \mathrm{H}_{2} \mathrm{O}, 2$; $\mathrm{ZnSO}_{4} \cdot 7 \mathrm{H}_{2} \mathrm{O}, 12 ; \mathrm{FeSO}_{4} \cdot 7 \mathrm{H}_{2} \mathrm{O}, 12 ; \mathrm{NaCl}, 12 ; \mathrm{KI}, 0.1 ; \mathrm{CoCl}_{2} \cdot 6 \mathrm{H}_{2} \mathrm{O}, 0.1$; $\mathrm{Na}_{2} \mathrm{MoO}_{4} \cdot 2 \mathrm{H}_{2} \mathrm{O}, 0.5 ; \mathrm{AlCl}_{3} \cdot 6 \mathrm{H}_{2} \mathrm{O}, 1$; and KF, 1 .

samples were put into RNase-free tubes and stored in liquid nitrogen for gene expression analysis and enzyme activity determination. The whole intestine from 12 fish (three fish per tank) was carefully removed then transferred to $2 \mathrm{~mL}$ sterile tubes and put into liquid nitrogen immediately for intestinal microbiota composition analysis.

All the experimental processes followed the Committee on the Ethics of Animal Experiments of Zhejiang University.

2.3. Sample Analysis. The liver and foregut supernatant was obtained according to Wang et al. [21]. The activities of superoxide dismutase (SOD), glutathione peroxidase (GSH-Px), catalase (CAT), and malondialdehyde (MDA) contents in the serum and liver were determined by the diagnostic reagent kits (catalog number: SOD, A001-3-2; GSH-
Px, A005-1-2; CAT, A007-1-1; MDA, A003-1-2) for fish (Nanjing Jiancheng Bioengineering Institute, Nanjing, China) following the manufacture's instruction.

2.4. Histological Observation. The liver and foregut samples for microscope observation were dehydrated by grades of ethanol and fixed in paraffin wax and then stained with hematoxylin and eosin (H\&E) after drying. Pictures were taken by the Olympus CX21 microscope. Foregut samples for scanning electron microscope (SEM) observation were washed in graded levels of ethanol and fixed in $1 \% \mathrm{OsO}_{4}$ for around two hours, before using different levels of ethanol to wash the samples gradually. The samples were subsequently dehydrated by liquid $\mathrm{CO}_{2}$ (Hitachi Model HCP-2), after which they were coated with gold-palladium (Hitachi Model E-1010 ion sputter). The SEM pictures were observed by Hitachi Model SU-8010.

2.5. RNA Extraction and $q P C R$. The total RNA of the liver and foregut samples (four replicates per treatment) were extracted by TaKaRa MiniBEST Universal RNA Extraction Kit (Takara, Japan). Then, cDNA was reversely transcribed by PrimeScript ${ }^{\mathrm{TM}}$ RT reagent Kit with gDNA Eraser (Perfect Real Time, Takara, Japan). The cDNA product was diluted with sterilized double-distilled water. The PCR primers of tnf $\alpha$ (tumor necrosis factor-alpha), il-1 $\beta$ (interleukin 1 beta), $n f-\kappa b$ (nuclear factor-kappa b), il-10 (interleukin-10), tof $\beta-1$ (transforming growth factor beta-1), Cu/Zn-sod (Cu/Znsuperoxide dismutase), and $g p x$ (glutathione peroxidase) were designed by Primer Premier 5.0 (Table 2). The qPCR process was set as $95^{\circ} \mathrm{C}$ for $30 \mathrm{~s}$ and 40 cycles of $95^{\circ} \mathrm{C}$ for $5 \mathrm{~s}$ followed by $60^{\circ} \mathrm{C}$ for $30 \mathrm{~s}$. $\beta$-Actin was used as the reference gene. SYBR Premix Ex Taq ${ }^{\mathrm{TM} I I}$ Kit (Takara, Japan) was used for real-time PCR. The relative gene expression level was calculated by the $2^{-\Delta \Delta C T}$ method [28].

2.6. DNA Extraction and Sequencing of Intestinal Microbiota. The E.Z.N.A. ${ }^{\circledR}$ Stool DNA Kit (D4015, Omega, Inc., USA) was used for the extraction of total DNA from the intestinal mucosa layer. The DNA was amplified using $16 \mathrm{~S}$ rDNA bacteria domain-specific primers for V4: 515F$806 \mathrm{R}$ region in a $25 \mu \mathrm{L}$ reaction system, with $12.5 \mu \mathrm{L}$ PCR premix, $25 \mathrm{ng}$ DNA template, $5 \mu \mathrm{L}$ primers, and PCR-grade water. The PCR process was set as follows: $98^{\circ} \mathrm{C}$ for $30 \mathrm{~s}$, 30 cycles of denaturation at $98^{\circ} \mathrm{C}$ for $10 \mathrm{~s}$, and $50^{\circ} \mathrm{C}$ for $30 \mathrm{~s}$ for annealing, followed by a final extension at $72^{\circ} \mathrm{C}$ for $10 \mathrm{~min}$. Then, $2 \%$ agarose gel electrophoresis was used to verify the PCR product. The PCR products were purified and quantified by LC-Bio Technology Co., Ltd., Hang Zhou, Zhejiang Province, China. Sequencing was performed on the NovaSeq PE250 platform.

Illumina NovaSeq platform (LC-Bio) was used for sample sequencing under the manufacturer's instruction. Paired-end reads were assigned to samples based on their unique barcode and truncated by cutting off the barcode and primer sequence. To obtain high-quality clean tags, fqtrim (v0.94) was used for filtering the raw reads. Chimera sequences were removed by Vsearch software (v2.3.4). Feature abundance was normalized by SILVA classifier. Chaol, 
TABle 2: Primer sequences of immune and antioxidative genes.

\begin{tabular}{|c|c|c|c|}
\hline Gene $^{1}$ & Nucleotide sequence & Size (bp) & GenBank or publication \\
\hline \multirow{2}{*}{$\operatorname{tnf} \alpha$} & Forward: GTCCTGCTGTTTGCTTGG & \multirow{2}{*}{154} & \multirow{2}{*}{ AY335443 } \\
\hline & Reverse: AATGGATGGCTGCCTTGG & & \\
\hline \multirow{2}{*}{$i l-1 \beta$} & Forward: CATCTGGAGGCGGTGAA & \multirow{2}{*}{231} & \multirow{2}{*}{ JQ973887 } \\
\hline & Reverse: CGGTTTTGGTGGGAGGA & & \\
\hline \multirow{2}{*}{$n f-\kappa b$} & Forward: AGCCCAAGGCACTCTAGACA & \multirow{2}{*}{154} & \multirow{2}{*}{ MK922543 } \\
\hline & Reverse: GTTCTGGGCAGCTGTAGAGG & & \\
\hline \multirow{2}{*}{$i l-10$} & Forward: CCCAGATAGAAGCCCAGGAT & \multirow{2}{*}{105} & \multirow{2}{*}{ MK922542 } \\
\hline & Reverse: AAACGATGATTTGGACACAGC & & \\
\hline \multirow{2}{*}{$\operatorname{tg} \beta-1$} & Forward: GGGTTTCCAACTTCGGC & \multirow{2}{*}{209} & \multirow{2}{*}{ Xue et al., 2008} \\
\hline & Reverse: TTGTGTCCGTGGAGCGT & & \\
\hline \multirow{2}{*}{ Cu/Zn-sod } & Forward: GTTGCCAAGATAGACATCAC & \multirow{2}{*}{800} & \multirow{2}{*}{ AJ000249.1 } \\
\hline & Reverse: TTAGACTCTCCTCGTTGC & & \\
\hline \multirow{2}{*}{$g p x$} & Forward: CAGGAGAACGGCAAGAAT & \multirow{4}{*}{264} & \multirow{4}{*}{ GU799605.1 } \\
\hline & Reverse: TTCCATTCACATCCACCTT & & \\
\hline \multirow{2}{*}{$\beta$-Actin } & Forward: ACCCAGATCATGTTCGAGACC & & \\
\hline & Reverse: ATGAGGTAGTCTGTGAGGTCG & & \\
\hline
\end{tabular}

${ }^{1}$ tnf $\alpha$ : tumor necrosis factor alpha;il-1 $\beta$ : interleukin 1 beta; $n f-\kappa b$ : nuclear factor-kappa b; il-10: interleukin-10; tgf $\beta$-1: transforming growth factor beta-1; Cul $\mathrm{Zn}$-sod: $\mathrm{Cu} / \mathrm{Zn}$-superoxide dismutase; $g p x$ : glutathione peroxidase.

TABLE 3: Effects of berberine inclusion in different diets on serum and liver antioxidative parameters of black sea bream ${ }^{1}$.

\begin{tabular}{|c|c|c|c|c|c|c|c|}
\hline \multirow{2}{*}{ Index $^{2}$} & \multirow{2}{*}{ Con } & \multirow{2}{*}{$\mathrm{HL}$} & \multirow{2}{*}{ ConB } & \multirow{2}{*}{ HLB } & \multicolumn{3}{|c|}{ Two-way ANOVA ( $P$ value $)$} \\
\hline & & & & & Berberine & Diet & Interaction \\
\hline \multicolumn{8}{|c|}{ Serum $(\mathrm{U} / \mathrm{mL})$} \\
\hline SOD & $164.16 \pm 7.68$ & $157.94 \pm 10.66$ & $177.56 \pm 2.02$ & $182.44 \pm 0.57$ & 0.046 & 0.925 & 0.451 \\
\hline GSH-Px & $183.78 \pm 12.07^{\mathrm{a}}$ & $151.12 \pm 7.21^{b}$ & $199.07 \pm 14.55^{\mathrm{a}}$ & $146.57 \pm 5.30^{\mathrm{b}}$ & 0.496 & 0.002 & 0.233 \\
\hline CAT & $2.86 \pm 0.75^{\mathrm{bc}}$ & $2.12 \pm 0.49^{c}$ & $4.80 \pm 0.76^{\mathrm{a}}$ & $3.59 \pm 0.16^{\mathrm{ab}}$ & 0.003 & 0.039 & 0.560 \\
\hline MDA & $12.16 \pm 0.90^{\mathrm{b}}$ & $14.38 \pm 0.61^{\mathrm{a}}$ & $12.31 \pm 0.72^{\mathrm{b}}$ & $13.12 \pm 0.18^{\mathrm{ab}}$ & 0.246 & 0.012 & 0.308 \\
\hline \multicolumn{8}{|c|}{ Liver (U/mg protein) } \\
\hline SOD & $325.32 \pm 26.78$ & $332.27 \pm 15.30$ & $361.95 \pm 16.05$ & $343.78 \pm 53.82$ & 0.229 & 0.770 & 0.516 \\
\hline GSH-Px & $7.68 \pm 0.98$ & $12.56 \pm 1.69$ & $10.40 \pm 0.29$ & $9.35 \pm 3.39$ & 0.869 & 0.231 & 0.085 \\
\hline CAT & $18.79 \pm 3.39^{\mathrm{ab}}$ & $18.38 \pm 2.88^{\mathrm{ab}}$ & $24.05 \pm 3.75^{\mathrm{a}}$ & $17.60 \pm 0.87^{\mathrm{b}}$ & 0.224 & 0.078 & 0.113 \\
\hline MDA & $2.89 \pm 0.58$ & $2.88 \pm 0.27$ & $3.73 \pm 0.71$ & $3.44 \pm 1.09$ & 0.138 & 0.734 & 0.751 \\
\hline
\end{tabular}

${ }^{1}$ Data are presented as the means $\pm \mathrm{SD}(n=3)$; values with different superscripts in the same row differ significantly $(P<0.05) .{ }^{2} \mathrm{SOD}$ : superoxide dismutase; MDA: malonaldehyde; GSH-Px: glutathione peroxidase; CAT: catalase.

observed species, Shannon, and Simpson are alpha diversity indices for analyzing the complexity of species diversity of all the samples calculated by QIIME2. Beta diversity was also calculated by QIIME2. Sequence alignment was performed by Blast, and feature sequences were annotated in the SILVA database. The diagrams were illustrated by the $\mathrm{R}$ package (v3.5.2).

2.7. Data Statistical Analysis. The data for antioxidative parameters, alpha diversity of the intestinal microbiota, and intestinal histological indices were tested for normality and homogeneity of variances by Kolmogorov-Smirnov and Levene's tests, respectively. Then, the data were subjected to one-way analysis of variance (ANOVA) by IBM
SPSS Statistics 20.0 (SPSS) using Duncan's multiple range tests, and different superscripts of mean values indicate the significant difference among different treatments based on one-way ANOVA analysis. Two-way ANOVA was used to determine the effects of berberine supplementation, dietary lipid level, and their interaction on these parameters. $P<$ 0.05 is considered significant. All data were expressed as mean values and standard deviations (SD).

\section{Result}

3.1. Antioxidative Parameters in the Serum and Liver. As shown in Table 3, fish fed the low-lipid diets (Con and ConB) had significantly higher serum GSH-Px activity than 


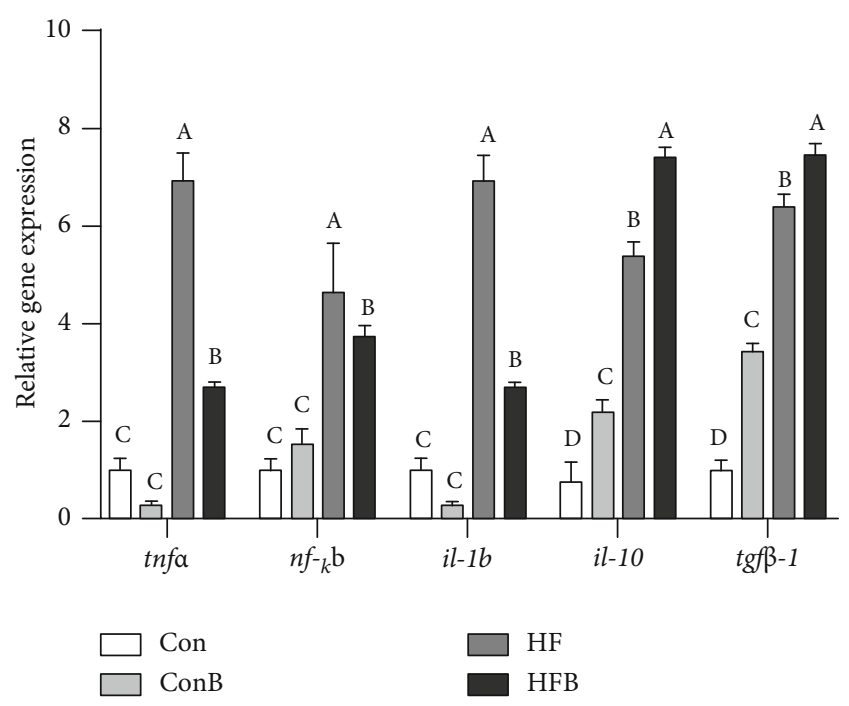

FIgURE 1: Relative expression levels of hepatic inflammation genes of black sea bream fed different diets $(n=3)$. Con: control group; ConB: control diet with $50 \mathrm{mg} / \mathrm{kg}$ berberine treatment; HL: highlipid group; HLB: high-lipid diet with $50 \mathrm{mg} / \mathrm{kg}$ berberine treatment.

TABle 4: Two-way ANOVA (P) analysis of gene expression of fish fed the experimental diets.

\begin{tabular}{lccc}
\hline Gene & Berberine & Diet & Interaction \\
\hline & & Liver & \\
$\operatorname{tnf\alpha }$ & 0.001 & $<0.001$ & 0.007 \\
$n f-\kappa b$ & 0.009 & $<0.001$ & $<0.001$ \\
$i l-1 \beta$ & $<0.001$ & $<0.001$ & $<0.001$ \\
Il-10 & $<0.001$ & $<0.001$ & 0.076 \\
$\operatorname{tgf} \beta-1$ & $<0.001$ & $<0.001$ & 0.007 \\
& & Intestine & \\
$\operatorname{tnf} \alpha$ & 0.450 & 0.838 & 0.689 \\
$n f-\kappa b$ & 0.224 & 0.137 & 0.337 \\
$\operatorname{tgf} \beta-1$ & 0.907 & 0.004 & 0.117 \\
$g p x$ & 0.962 & 0.247 & 0.422 \\
Cu/Zn-sod & 0.160 & 0.107 & 0.134 \\
\hline
\end{tabular}

that of the HL and HLB groups $(P<0.05)$. Berberine supplementation in the control and high-lipid diets significantly improved serum CAT activity $(P<0.05)$. The highest serum MDA content was found in fish fed the HL diet. In the liver, the highest CAT activity was in fish fed the ConB diet, while SOD and GSH-Px activities, as well as MDA content in the liver, showed no significant differences among the different groups $(P>0.05)$. Based on two-way ANOVA analysis, serum GSH-Px activity was significantly influenced by dietary lipid level, and serum CAT activity was significantly affected by berberine supplementation and dietary lipid level $(P<0.05)$.

3.2. Gene Expression in the Liver and Foregut. As shown in Figure 1, the relative expression levels of $n f-k b, i l-1 \beta, \operatorname{tnf} \alpha$, il-10, and $\operatorname{tg} \beta-1$ were significantly upregulated in the liver

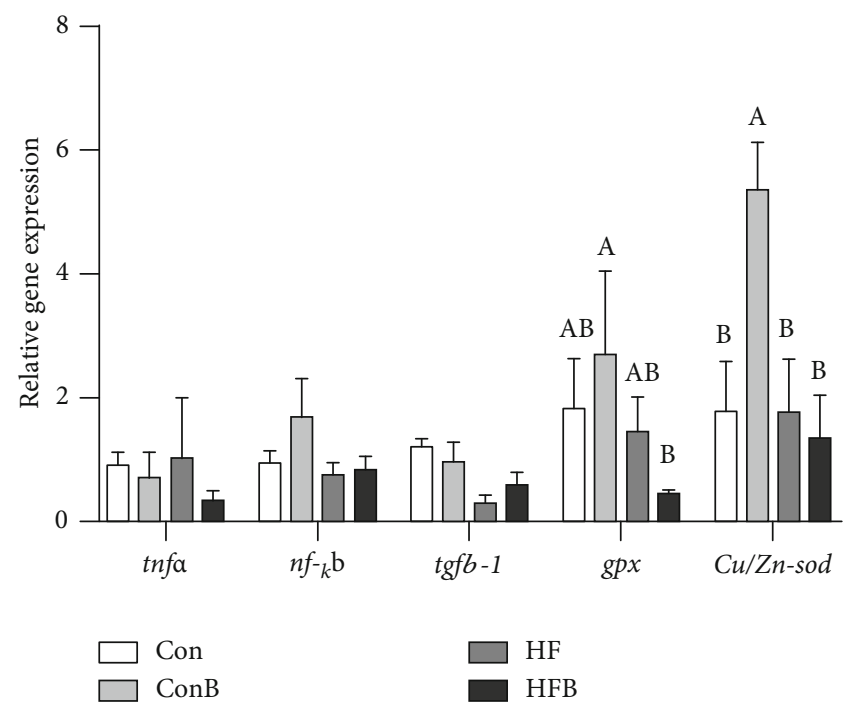

FIgURE 2: Relative expression levels of foregut inflammation and antioxidative genes of black sea bream fed different diets $(n=3)$. Con: control group; ConB: control diet with $50 \mathrm{mg} / \mathrm{kg}$ berberine treatment; HL: high-lipid group; HLB: high-lipid diet with $50 \mathrm{mg} /$ $\mathrm{kg}$ berberine treatment.

of fish fed HL diet compared with that of the Con group, while berberine supplementation in high-lipid diet significantly downregulated $t n f \alpha, n f-\kappa b$, and $i l-1 \beta$ expressions $(P<0.05)$. Berberine supplementation in normal and highlipid diets significantly upregulated $i l-10$ and $\operatorname{tg} f \beta-1$ expressions in the liver $(P<0.05)$. Based on the two-way analysis (Table 4 ), all these genes were affected by both dietary lipid level and berberine supplementation significantly, and significant interactions between berberine and dietary lipid level were observed in all these gene expression levels (except for il-10).

In the foregut (Figure 2), $n f-\kappa b, \operatorname{tnf} \alpha$, and $\operatorname{tg} f \beta-1$ expression levels in the different groups showed no significant differences $(P>0.05)$. Fish fed the ConB diet had a significantly upregulated expression level of $\mathrm{Cu} / \mathrm{Zn}$-sod than that of the other three groups $(P<0.05)$. Based on the two-way analysis (Table 4$)$, these genes were not significantly influenced by berberine supplementation or dietary lipid level, and no significant interaction was observed.

3.3. Intestinal Morphology. As shown in Figure 3, the intestinal villus of the fish fed Con, ConB, and HLB diets were intact and neat, whereas fish fed the HL diet had relatively sparse intestinal villus, fewer mucosal folds along with expanded lamina propria, and decreased muscular mucosae. The HL group showed the shortest villus length but the highest number of goblet cells among the treatments (Table 5). Based on two-way ANOVA, villus length was significantly influenced by the dietary lipid level, and significant interaction between dietary lipid level and berberine supplementation was observed in villus length $(P<0.05)$.

According to the SEM result in Figure 4 and Table 5, berberine supplementation in the normal diet significantly increased intestinal microvillus density $(P<0.05)$. Besides, 

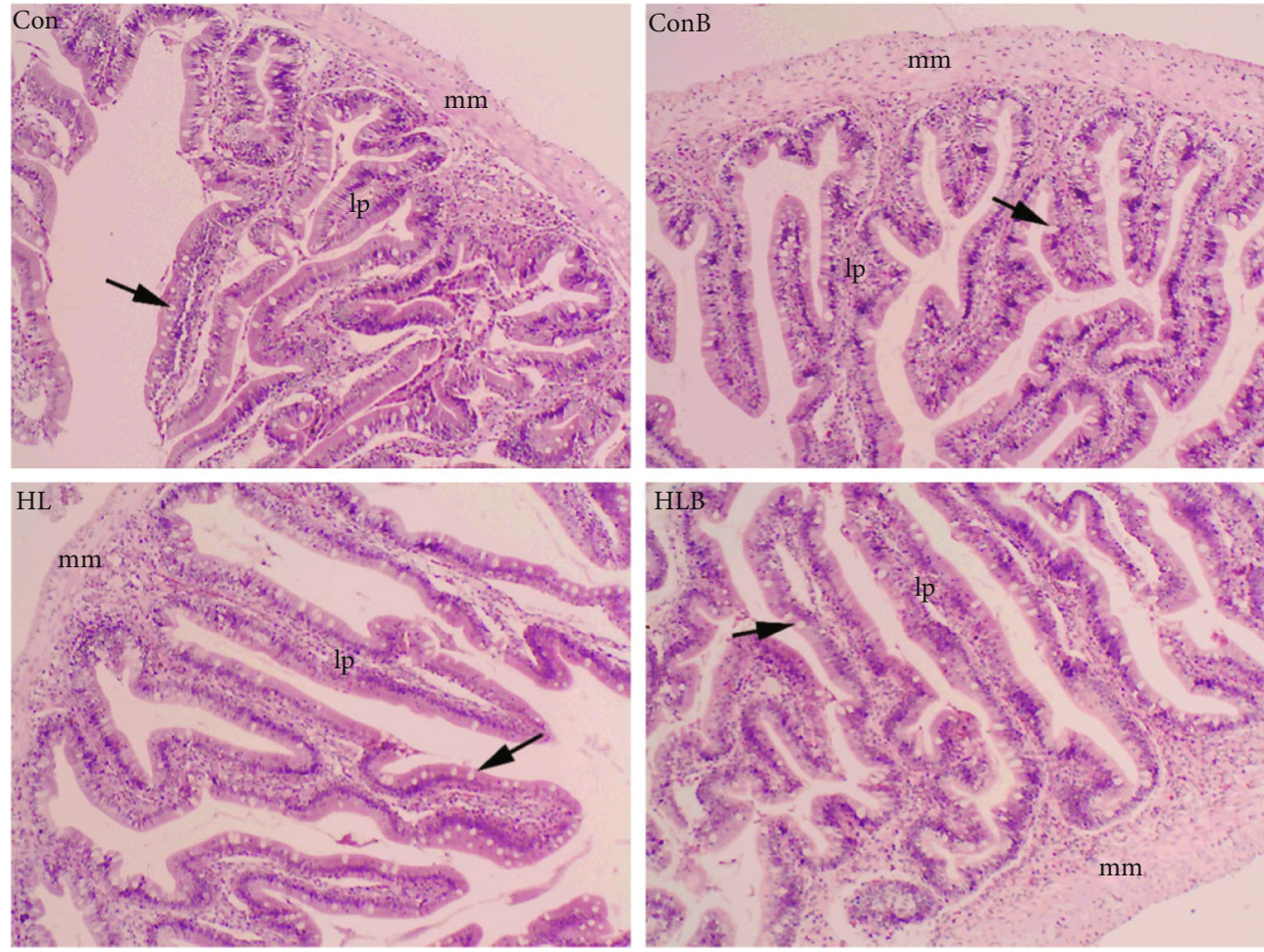

FIGURE 3: Intestinal morphology $(\times 100)$ of black sea bream subjected to different treatments, hematoxylin-eosin staining (HE). Con: control group; ConB: control diet with $50 \mathrm{mg} / \mathrm{kg}$ berberine treatment; HL: high-lipid group; HLB: high-lipid diet with $50 \mathrm{mg} / \mathrm{kg}$ berberine treatment. Black arrow shows the goblet cell scattered among the enterocytes. The intestinal villus of fish in berberine diets was neat and intact. The intestinal villus high uniformity and integrity were impaired in the HF group. The epithelial cells disorganized with increased width of the lamina propria; increased goblet cell (black arrow) count, and decreased muscularis mucosae, and mucosal folds were observed in fish fed HF diet. lp: lamina propria, mm: muscularis mucosae.

TABLE 5: Effects of berberine inclusion in different diets on intestinal villus length and goblet cell number of black sea bream ${ }^{1}$.

\begin{tabular}{|c|c|c|c|c|c|c|c|}
\hline \multirow{2}{*}{ Index } & \multirow{2}{*}{ Con } & \multirow{2}{*}{$\mathrm{HL}$} & \multirow{2}{*}{ ConB } & \multirow{2}{*}{ HLB } & \multicolumn{3}{|c|}{ Two-way ANOVA ( $P$ value) } \\
\hline & & & & & Berberine & Diet & Interaction \\
\hline Villus length (um) & $822 \pm 236^{\mathrm{a}}$ & $520 \pm 139^{b}$ & $680 \pm 139^{a}$ & $695 \pm 156^{\mathrm{a}}$ & 0.756 & 0.013 & 0.006 \\
\hline Goblet cells (per villi) & $17.28 \pm 2.44^{\mathrm{ab}}$ & $20.71 \pm 3.06^{\mathrm{a}}$ & $13.57 \pm 3.41^{\mathrm{b}}$ & $18.28 \pm 3.90^{\mathrm{ab}}$ & 0.075 & 0.174 & 0.772 \\
\hline Microvillus density $\left(\mu \mathrm{m}^{2}\right)$ & $85.50 \pm 8.58^{\mathrm{b}}$ & $113.00 \pm 11.49^{\mathrm{a}}$ & $109.00 \pm 8.12^{\mathrm{a}}$ & $119.50 \pm 4.43^{\mathrm{a}}$ & 0.004 & 0.001 & 0.070 \\
\hline
\end{tabular}

${ }^{1}$ Data are presented as the means $\pm \mathrm{SD}(n=3)$; values with different superscripts in the same row differ significantly $(P<0.05)$.

dietary lipid level and berberine supplementation influenced intestinal microvillus density significantly based on two-way ANOVA analysis $(P<0.05)$.

3.4. Intestinal Microbiota. As shown in Table 6, observed species and Chaol were used to estimate community richness, while Simpson's and Shannon's indices were used to determine community diversity. Shannon's indices of the HL group were significantly lower than that of the HLB group, indicating berberine supplementation in the HL diet improved the diversity of intestinal microbiota significantly, whereas Simpson's indices showed no significant difference among different treatments. Observed species and Chaol in the HL group were significantly lower than those of the Con group, indicating that high-lipid treatment reduced richness in the intestinal microbiota of black sea bream.
A sum of 144 operational taxonomic units (OTUs) was shared among the four treatments. Fish fed the Con diet had the highest unique OTUs (195), followed by those from the fish fed the HLB (175), ConB (134), and HL (129) diets (Figure 5).

3.5. Community Composition of Intestinal Microbiota. Proteobacteria was the most abundant phylum in all treatments, followed by Cyanobacteria, Firmicutes, Bacteroidetes, and Acidobacteria (Table 7). The fish fed HL diet had a significantly higher abundance of Bacteroidetes than that of the Con and HLB groups. Fish fed HLB and Con diets had similar intestinal microbiota composition in phylum (Figure 6(a)). Pseudomonas, Ralstonia, Chloroplast_unclassified, Brevundimonas, Weissella, Hydrogenophaga, Caulobacter, Sphingomonas, and Novosphingobium were the top 10 

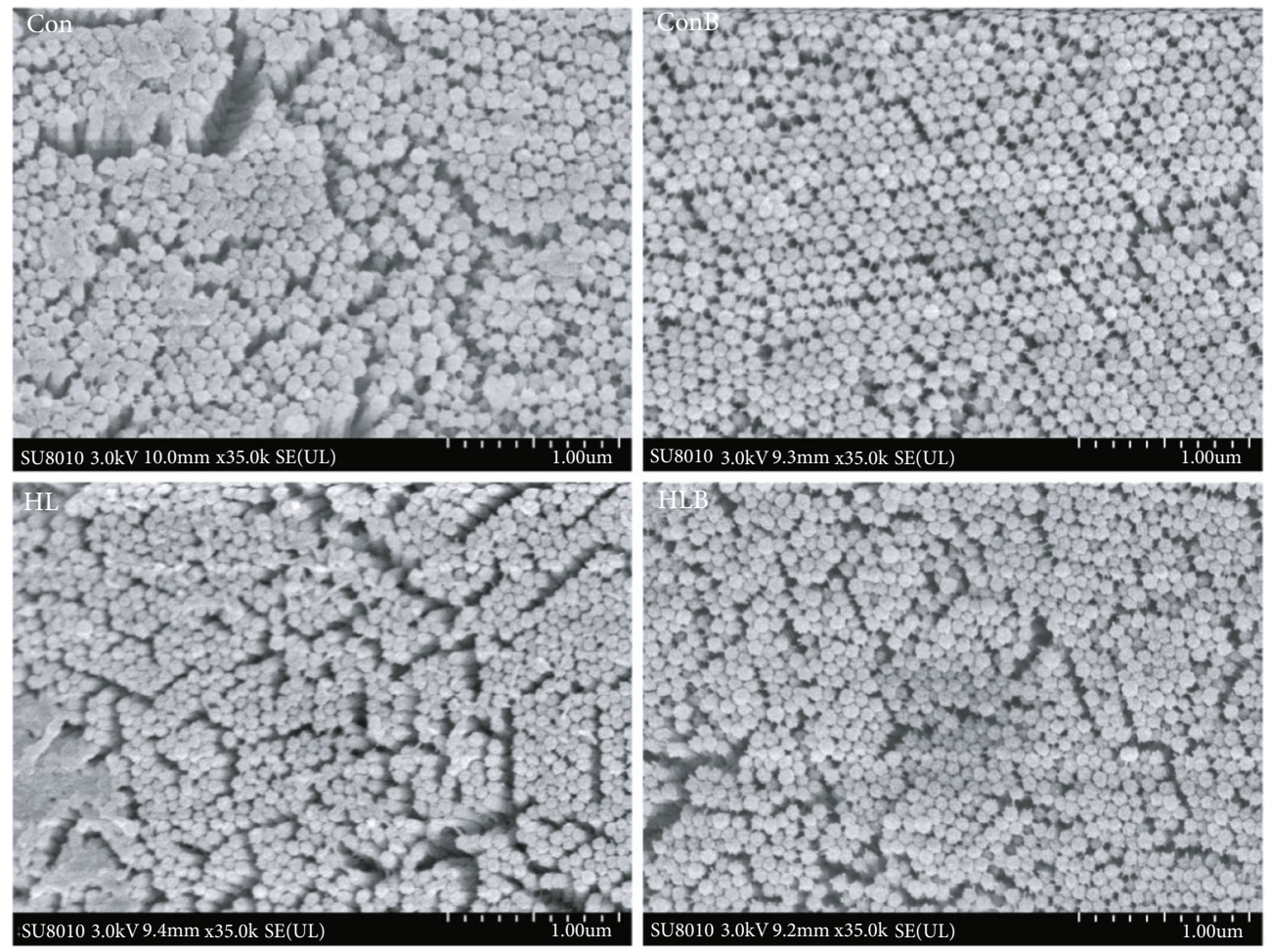

FIGURE 4: Scanning electron microscopy of villus structure changes in anterior gut of black sea bream $(\times 3500$ magnifications). Con: control group; ConB: control diet with $50 \mathrm{mg} / \mathrm{kg}$ berberine treatment; HL: high-lipid group; HLB: high lipid diet with $50 \mathrm{mg} / \mathrm{kg}$ berberine treatment.

Table 6: Alpha diversity index of intestine microbiota of black sea bream fed different experimental diets ${ }^{1}$.

\begin{tabular}{|c|c|c|c|c|c|c|c|}
\hline \multirow{2}{*}{ Index } & \multirow{2}{*}{ Con } & \multirow{2}{*}{$\mathrm{HL}$} & \multirow{2}{*}{ ConB } & \multirow{2}{*}{ HLB } & \multicolumn{3}{|c|}{ Two-way ANOVA ( $P$ value) } \\
\hline & & & & & Berberine & Diet & Interaction \\
\hline Observed species & $256.00 \pm 28.59^{\mathrm{a}}$ & $183.33 \pm 21.94^{\mathrm{b}}$ & $222.0 \pm 10.41^{\mathrm{ab}}$ & $242.33 \pm 5.81^{\mathrm{ab}}$ & 0.529 & 0.205 & 0.040 \\
\hline Shannon & $4.25 \pm 0.10^{\mathrm{ab}}$ & $3.94 \pm 0.22^{\mathrm{c}}$ & $3.99 \pm 0.17^{\mathrm{bc}}$ & $4.27 \pm 0.07^{\mathrm{a}}$ & 0.835 & 0.933 & 0.094 \\
\hline Simpson & $0.88 \pm 0.11$ & $0.85 \pm 0.03$ & $0.85 \pm 0.03$ & $0.89 \pm 0.01$ & 1.000 & 0.761 & 0.155 \\
\hline Chaol & $258.06 \pm 29.53^{\mathrm{a}}$ & $183.92 \pm 22.41^{\mathrm{b}}$ & $222.62 \pm 10.29^{\mathrm{ab}}$ & $243.53 \pm 5.87^{\mathrm{ab}}$ & 0.552 & 0.209 & 0.040 \\
\hline
\end{tabular}

${ }^{1}$ Data are presented as the means $\pm \mathrm{SD}(n=3)$; values with different superscripts in the same row differ significantly $(P<0.05)$.

genera in the intestinal microbiota of black sea bream fed the different diets (Table 7). At the genus level, fish fed Con and HLB diets had a similar intestinal composition, while the ConB and HL groups shared the close bacterial genera composition (Figure 7). Fish fed the HL diet had a significantly lower abundance of Pseudomonas and Hydrogenophaga than that of fish fed the HLB diet $(P<0.05)$ (Table 7$)$. And significant interaction between berberine supplementation and diet was observed in the abundances of Pseudomonas, Ralstonia, and Hydrogenophaga.

Linear discriminant analysis (LDA) effect size (LEfSe) analysis was used to estimate the relative bacterial abundance in the taxon of black sea bream fed different diets. Fish fed Con, ConB, HL, and HLB diets had 16, 2, 9, and 16 significantly enriched taxa than the other treatments, respectively (Figure 8). These 43 taxa were classified from 5 orders, 7 families, 13 genera, and 18 species. Biomarkers of the Con group included order Bacillales, family Bacillacease, and genus Anaerotruncus, Pseudoxanthomonas, Eisenbergiella, Blautia, Oceanobacillus, and Clostridium. The biomarker of the ConB group included genus Comamonas. Biomarkers of the HL group included order Flavobacteriales, Actinobacteria-unclassified, family Weeksellaceae and Actinobacteria-unclassified, genus Actinobacteria-unclassified and Elizabethkingia. Biomarkers of the HL group included order Pseudomonadales and Enterobacterales, family Pseudomonadaseae, Moraxellaceae, Planococcaceae, and Enterobacteriaceae, and genus Pseudomonas, Psychrobacter, Ruegeria, and Lysinibacillus.

\section{Discussion}

Massive hepatic lipid accumulation and increased liver $n f$ $\kappa b$, il-1 $\beta$, and $t n f \alpha$ expression levels caused by high dietary lipid have been reported in black sea bream [29, 30]. Normally, too much lipid deposition in the liver results in 


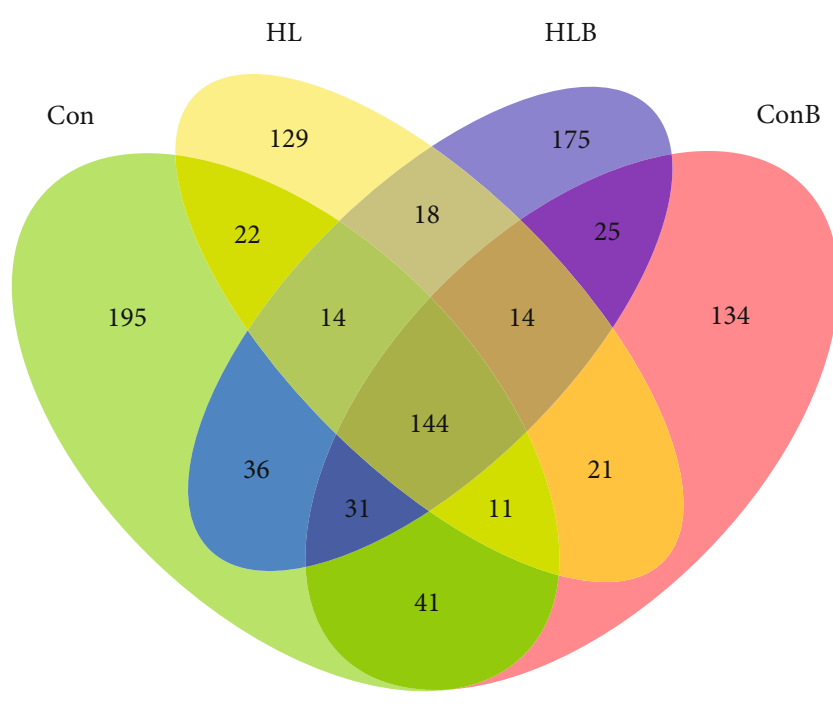

FIGURE 5: Venn diagram showing OTUs analysis of black sea bream intestinal microbiota fed different experimental diets $(n=3$ ). Con: control group; ConB: control diet with $50 \mathrm{mg} / \mathrm{kg}$ berberine treatment; HL: high-lipid group; HLB: high-lipid diet with $50 \mathrm{mg} /$ $\mathrm{kg}$ berberine treatment.

endoplasmic reticulum stress, which may promote proinflammatory factor (like $i l-1 \beta$, and $t n f \alpha$ ) expressions [29-31]. High-lipid diet causing liver inflammation has been reported in many fish species, like blunt snout bream [22] and turbot (Scophthalmus maximus) [32]. In the present study, berberine supplementation in high-lipid diet significantly downregulated the expression levels of these proinflammatory factors, and berberine supplementation in normal and high-lipid diets also promoted antiinflammatory cytokine ( $i l-10$ and $\operatorname{tg} f \beta-1$ ) expression levels. These results indicate that berberine could reduce liver inflammation induced by high-lipid diet. The antiinflammation function may be attributed to the ability of berberine to alleviate liver lipid accumulation as reported by Wang et al. [19]. Similarly, other hepatic lipid-lowering additives also showed an anti-inflammation effect in black sea bream, such as L-carnitine and bile acid [29], choline [30] and fenofibrate [31]. Studies in mice found that berberine alleviated nonalcoholic steatohepatitis by inhibiting phosphoinositide 3 -kinase (PI3K), NF- $\kappa \mathrm{B}$, and IL-8 in the cysteine $\mathrm{X}$ cysteine ligand 12/cysteine $\mathrm{X}$ cysteine receptor 4 (CXCL12/CXCR4) inflammatory signaling pathway [33], or by activating AMPK signaling and decreasing the TLR4/ NF- $\kappa$ B-p65 signaling pathway [34]. In addition, the antiinflammatory effect of berberine has been reported in 3T3L1 adipocytes [35] and visceral adipose tissue [36]. However, in the present study, $\operatorname{tnf} \alpha, n f-\kappa b$, and $\operatorname{tg} f \beta-1$ expression levels in the foregut were not significantly influenced by high-lipid diet or berberine supplementation. The rebalanced intestinal microbiota by berberine supplementation might be a contributing factor since the intestinal immune response is highly related to microbiota dysbiosis [24]. However, highlipid diet upregulated intestinal $n f-\kappa b$ [30], $\operatorname{nf} \alpha$ [29], and $i l-1 \beta$ [31] expressions in black sea bream in the previous studies. A study in blunt snout bream observed that $50 \mathrm{mg}$ / $\mathrm{kg}$ berberine supplementation in high-lipid and highcarbohydrate diets significantly inhibited intestinal tnfo and il-6 expressions [17]. These inconsistencies could be due to the differences in basal diet, fish species and size, the segment of intestine for analysis, and environmental conditions.

In the present study, high-lipid diets (HL and HLB) reduced serum GSH-Px activity significantly, while berberine supplementation in normal and high-lipid diets improved serum CAT activity significantly. Berberine was proven to quench superoxide anions and nitric oxide effectively [6], and it can reduce ROS by the p38 MAPK pathway [5]. Previously, berberine supplementation in high-starch diet enhanced serum GSH-Px activity before and after ammonia stress challenge in black sea bream $[37,38]$. Chen et al. [25] observed that $50 \mathrm{mg} / \mathrm{kg}$ berberine supplementation in a HL diet reduced hepatic MDA, lipid peroxide, and protein carbonyl contents of blunt snout bream through elevating SOD activity and total sulfhydryl levels. Another study in blunt snout bream found that the HF (15\% fat) diet damaged mitochondria normal structure and induced more ROS production in the liver, while berberine supplementation reduced hepatic MDA content along with increased glutathione (GSH) activity via upregulating Sirtuin 3 expression and increasing the complex I and II activities [22]. Xu et al. [39] also reported that berberine $(50 \mathrm{mg} / \mathrm{kg})$ supplementation in normal and high-fat diets improved hepatic CAT and SOD activities with a decreased MDA content regardless of different feeding modes. Chen et al. [27] demonstrated that berberine could eliminate hepatic ROS production and reduce DNA damage caused by the high-cholesterol diet of zebrafish. However, these studies did not determine the antioxidative parameters in serum, whereas the present study found that berberine supplementation enhanced serum antioxidative status more significantly than that of the liver. This could be because berberine was widely distributed in the organs and tissues after oral administration, and it enhanced the whole-body antioxidative status. Interestingly, the significantly upregulated expression of $\mathrm{Cu} / \mathrm{Zn}$-sod in the foregut was only observed in the ConB group, and hepatic CAT activity of the ConB group was significantly higher than the other groups, indicating that the antioxidative effect of berberine might be influenced by dietary lipid level.

However, intestinal morphology was affected by the high-lipid diet in the present study, such as a reduced muscular mucosae thickness, villus length, and expanded lamina propria. The intestinal physical barrier directly determines the absorption capacity of nutrients, and its integrity is critical for intestinal health [40]. A high-fat diet may disrupt the gut barrier function [41]. Endotoxin, mainly are lipopolysaccharides, can damage the intestinal structure and increase intestinal permeability, as well as trigger inflammation response and change intestinal microbial community [37, 38]. Thus, the high-fat diet might induce more endotoxin and cause intestinal structural changes in the present study, whereas berberine supplementation in high-lipid diet alleviated the pathological changes caused by the high-lipid diet. It has been proved that berberine inhibits the intestinal secretory response of bacterial enterotoxin, regulates 
TABle 7: Top 5 phylum and top 10 genus of microbiota in black sea bream fed different experimental diets ${ }^{1}$.

\begin{tabular}{|c|c|c|c|c|c|c|c|}
\hline \multirow{2}{*}{$\begin{array}{l}\text { Berberine } \\
\text { Diet }\end{array}$} & \multirow{2}{*}{ Con } & \multirow{2}{*}{$\mathrm{HL}$} & \multirow{2}{*}{ ConB } & \multirow{2}{*}{ HLB } & \multicolumn{3}{|c|}{ Two-way ANOVA ( $P$ value) } \\
\hline & & & & & Berberine & Diet & Interaction \\
\hline \multicolumn{8}{|l|}{ Phylum } \\
\hline Proteobacteria & $80.22 \pm 0.27$ & $71.30 \pm 6.25$ & $78.22 \pm 6.29$ & $78.27 \pm 6.12$ & 0.615 & 0.378 & 0.373 \\
\hline Cyanobacteria & $10.91 \pm 1.09$ & $19.71 \pm 4.72$ & $15.86 \pm 5.82$ & $10.47 \pm 2.11$ & 0.601 & 0.676 & 0.109 \\
\hline Firmicutes & $7.38 \pm 0.96$ & $6.75 \pm 4.23$ & $4.21 \pm 1.03$ & $9.78 \pm 4.04$ & 0.979 & 0.346 & 0.244 \\
\hline Bacteroidetes & $0.31 \pm 0.05^{\mathrm{b}}$ & $0.97 \pm 0.27^{\mathrm{a}}$ & $0.55 \pm 0.11^{\mathrm{ab}}$ & $0.43 \pm 0.07^{\mathrm{b}}$ & 0.365 & 0.110 & 0.032 \\
\hline Acidobacteria & $0.60 \pm 0.07$ & $0.49 \pm 0.22$ & $0.54 \pm 0.02$ & $0.52 \pm 0.01$ & 0.893 & 0.594 & 0.728 \\
\hline \multicolumn{8}{|l|}{ Genus } \\
\hline Pseudomonas & $34.50 \pm 3.78^{\mathrm{a}}$ & $17.72 \pm 2.87^{b}$ & $21.05 \pm 3.01^{\mathrm{b}}$ & $34.86 \pm 2.59^{\mathrm{a}}$ & 0.596 & 0.646 & 0.001 \\
\hline Ralstonia & $14.03 \pm 3.38$ & $27.15 \pm 7.46$ & $28.72 \pm 7.24$ & $12.27 \pm 3.44$ & 0.988 & 0.779 & 0.033 \\
\hline Chloroplast_unclassified & $10.89 \pm 1.88$ & $19.70 \pm 8.17$ & $15.84 \pm 10.11$ & $10.45 \pm 3.69$ & 0.600 & 0.676 & 0.109 \\
\hline Brevundimonas & $11.68 \pm 0.70$ & $9.80 \pm 3.78$ & $9.87 \pm 1.91$ & $10.63 \pm 1.25$ & 0.772 & 0.737 & 0.440 \\
\hline Weissella & $4.34 \pm 0.97$ & $3.85 \pm 2.53$ & $1.78 \pm 0.72$ & $7.04 \pm 3.76$ & 0.896 & 0.340 & 0.257 \\
\hline Hydrogenophaga & $4.60 \pm 0.53^{\mathrm{ab}}$ & $2.68 \pm 0.94^{\mathrm{b}}$ & $3.23 \pm 0.70^{\mathrm{ab}}$ & $5.44 \pm 0.50^{\mathrm{a}}$ & 0.343 & 0.837 & 0.017 \\
\hline Caulobacter & $2.48 \pm 0.67$ & $2.47 \pm 0.72$ & $2.68 \pm 0.66$ & $1.17 \pm 0.14$ & 0.385 & 0.238 & 0.244 \\
\hline Sphingomonas & $1.49 \pm 0.02$ & $1.29 \pm 0.29$ & $1.34 \pm 0.27$ & $1.22 \pm 0.04$ & 0.617 & 0.457 & 0.850 \\
\hline Novosphingobium & $0.88 \pm 0.17$ & $0.81 \pm 0.13$ & $1.02 \pm 0.23$ & $0.82 \pm 0.07$ & 0.635 & 0.432 & 0.706 \\
\hline
\end{tabular}

${ }^{1}$ Data are presented as the means \pm SD $(n=3)$; values with different superscripts in the same row differ significantly $(P<0.05)$.

intestinal motility, and restores intestinal barrier function [42]. Berberine alleviated sparsely arranged intestinal mucosal villi and decreased gap junctions in nonalcoholic fatty liver disease (NAFLD) rats $[37,38]$. In the present study, berberine supplementation in the normal diet increased intestinal microvillus density significantly, and both dietary lipid level and berberine were contributing factors that influenced microvillus density. Likewise, blunt snout bream fed high-lipid or high-carbohydrate diet caused a decrease of microvilli length, but berberine supplementation increased it significantly [17]. In this study, black sea bream fed the HL diet exhibited increased goblet cells count, which might be attributed to the alteration in mucin dynamic [43]. Generally, high-lipid diet triggers more liberation of mucin in the intestine to relieve the intestinal inflammation process, which induces a vast secretion of molecules by the host to maintain the intestinal barrier function and a viscoelastic protective layer composed of mucins secreted by goblet cells [24]. Thus, the increased goblet cells in HL treatment might be compensation for impaired intestinal immunity. In accordance with the present study, increased mucus production and goblet cells in the intestine induced by a high-fat diet was reported in zebrafish [24]. Studies in Atlantic salmon (Salmo salar L.) and grass carp also demonstrated that intestinal inflammation is correlated to increased goblet cell numbers $[44,45]$. In addition, the intestinal morphology alteration might be related to the changes in the gut microbiota. Germ-free rodents had fewer goblet cells with a smaller size compared with conventionally raised mice. Besides, the mucus layer in conventionally raised mice was twice in thickness as that of germ-free rodents [43].
Host gut microbiota is influenced by the proportion and composition of dietary lipids [46]. In the present study, high-lipid diet reduced the richness and diversity of gut microbiota. A similar result was also observed in rice field eel (Monopterus albus) [23]. Li et al. [12] pointed out that healthy fish has a relatively higher bacterial diversity than diseased fish and reduced microbiota richness is a sign of some harmful conditions. For example, germ-free fish suffered impaired nutrient absorption, immunosuppression, intestinal epithelial cell dysfunction, altered metabolism, and weakened barriers against pathogens [47]. Studies in humans found that reduced microbial diversity might link to some diseases, such as type-2 diabetes and obesity [48]. Similarly, a high soybean meal diet also reduced intestinal microbiota in yellow drum (Nibea albiflora) [49]. In the present study, berberine supplementation in high-lipid diet improved the richness and diversity of the intestinal microbiota, indicating that berberine could rebalance intestinal microbiota impaired by high-lipid diet. In addition, Pan et al. [3] reported that compared with the control group, alpha diversity was decreased during berberine feeding, while an opposite trend showed after berberine feeding stopped in the berberine group, indicating that berberine might maintain the stability of gut microbiota of grass carp.

In the only study performed on black sea bream, Firmicutes and Proteobacteria were the dominant phyla in the intestine [50]. But the present study found that the main intestinal phyla were Proteobacteria and Cyanobacteria in black sea bream. Intestinal microbiota can be affected by certain factors, including genetic background, living environment, and diet ([51]). Proteobacteria being the main phylum in the intestine is inconsistent with the results in 

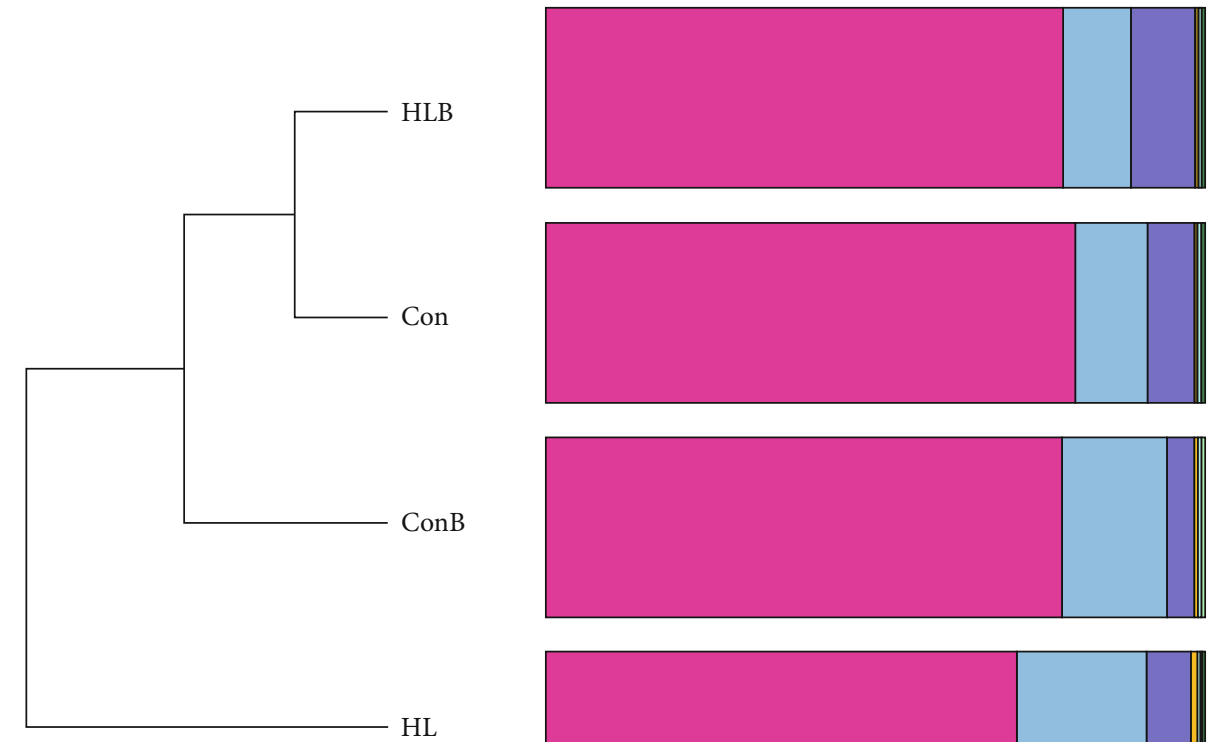

Proteobacteria

Cyanobacteria

- Firmicutes

Bacteroidetes

Acidobacteria

Actinobacteria

Planctomycetes

- Gemmatimonadetes

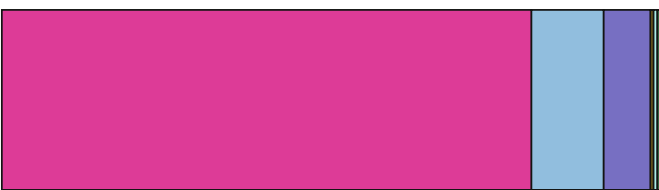

Verrucomicrobia

- Patescibacteri

- Chloroflexi

- Deinococcus-Thermus

- Epsilonbacteraeot

- Chlamydiae

Spirochaetes

- Nitrospirae

Fusobacteria

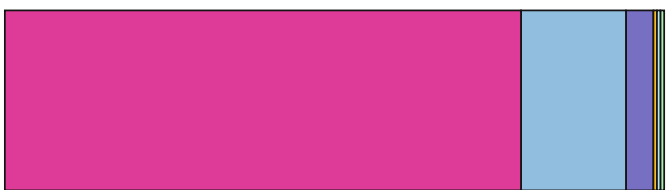

Deferribacteres

HL
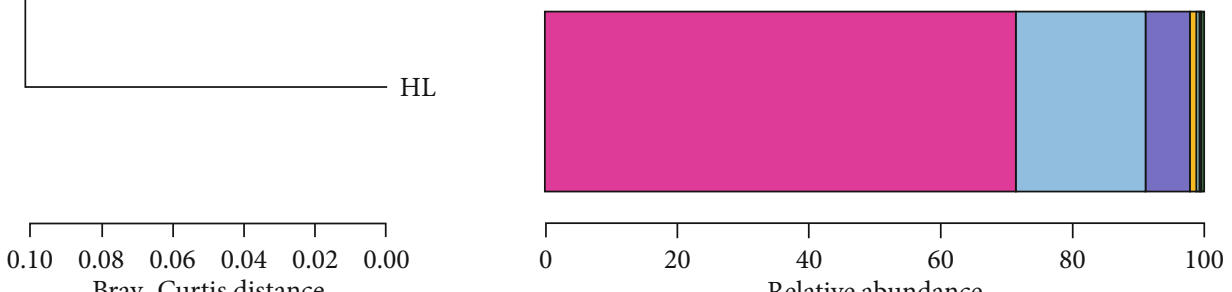

Bray-Curtis distance

(a)
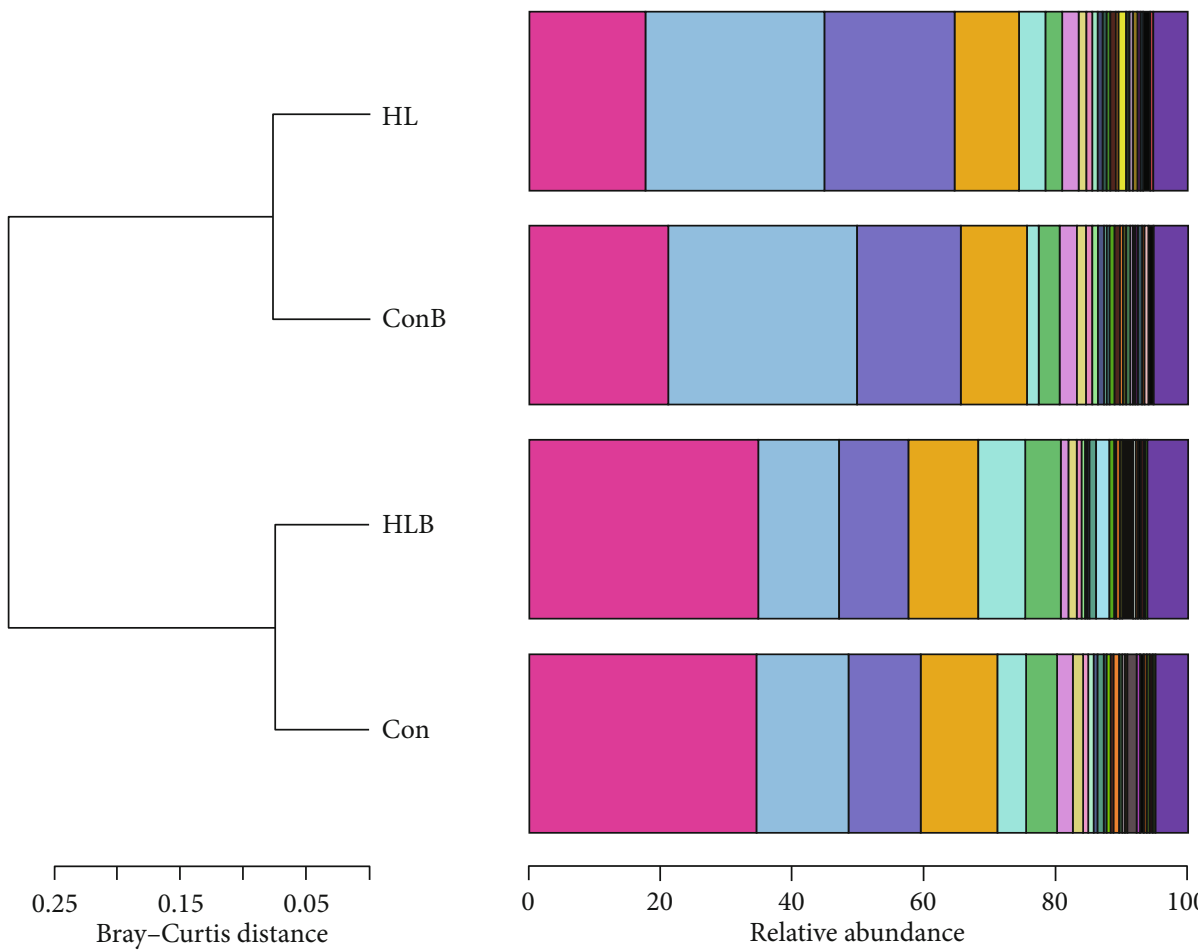

- Pseudomonas

- Chloroplast-unclassified

- Brevundimonas

al Weissella

- Hydrogenophaga

- Caulobacter

- - Sphingomonas

- Novosphingobium

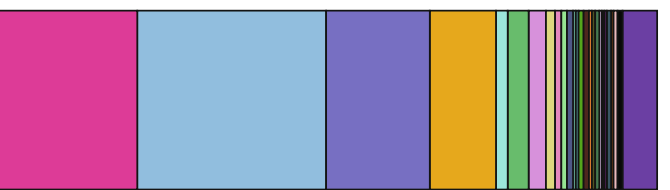

- Achromobacter

- Pleomorphomonadaceae_unclassified

- Devosia

Psychrobacter

- Methyloversatilis

- Bradyrhizobium

- Lactobacillus

- Lactobacillus

- Dechloromonas

- Stenotrophomon

Clostridium sensus

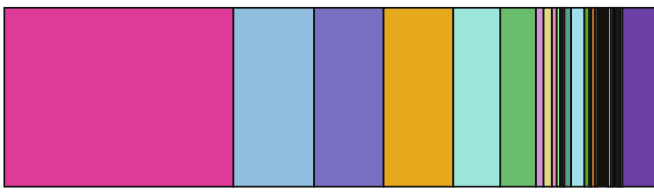

- Herbaspirillum

- Mitochondria_unclassified

Lutispora

Acinetobacter

- Subgroup_7_unclassified

- Vibrio

Rhodopseudomona

Rhodopseudomonas

- Actinobacteria_unclassified

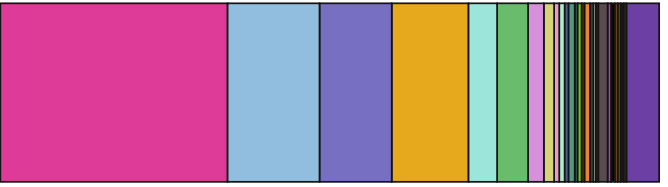

Mesorhizobium

Others Con

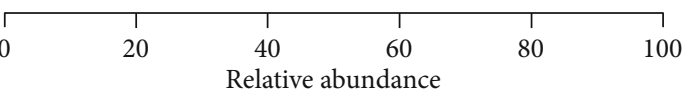

(b)

Figure 6: Taxonomy classification of reads at phylum (a) and genus (b) levels. Top 30 most abundant bacterial phyla and genera are presented in the figure, and unshown phyla and genera were assigned as "Others" $(n=3)$. Con: control group; ConB: control diet with $50 \mathrm{mg} / \mathrm{kg}$ berberine treatment; HL: high-lipid group; HLB: high-lipid diet with $50 \mathrm{mg} / \mathrm{kg}$ berberine treatment.

other aquatic animals, such as swimming carb [10], zebrafish [24], white leg shrimp (Penaeus vannamei) [11], channel catfish (Ictalurus punctatus) [52], and blunt snout bream [17].
In the present study, fish fed the HL diet had a significantly higher community of Bacteroidetes in the intestine, while berberine supplementation in high-lipid diet reduced the abundance of Bacteroidetes significantly. Similarly, 


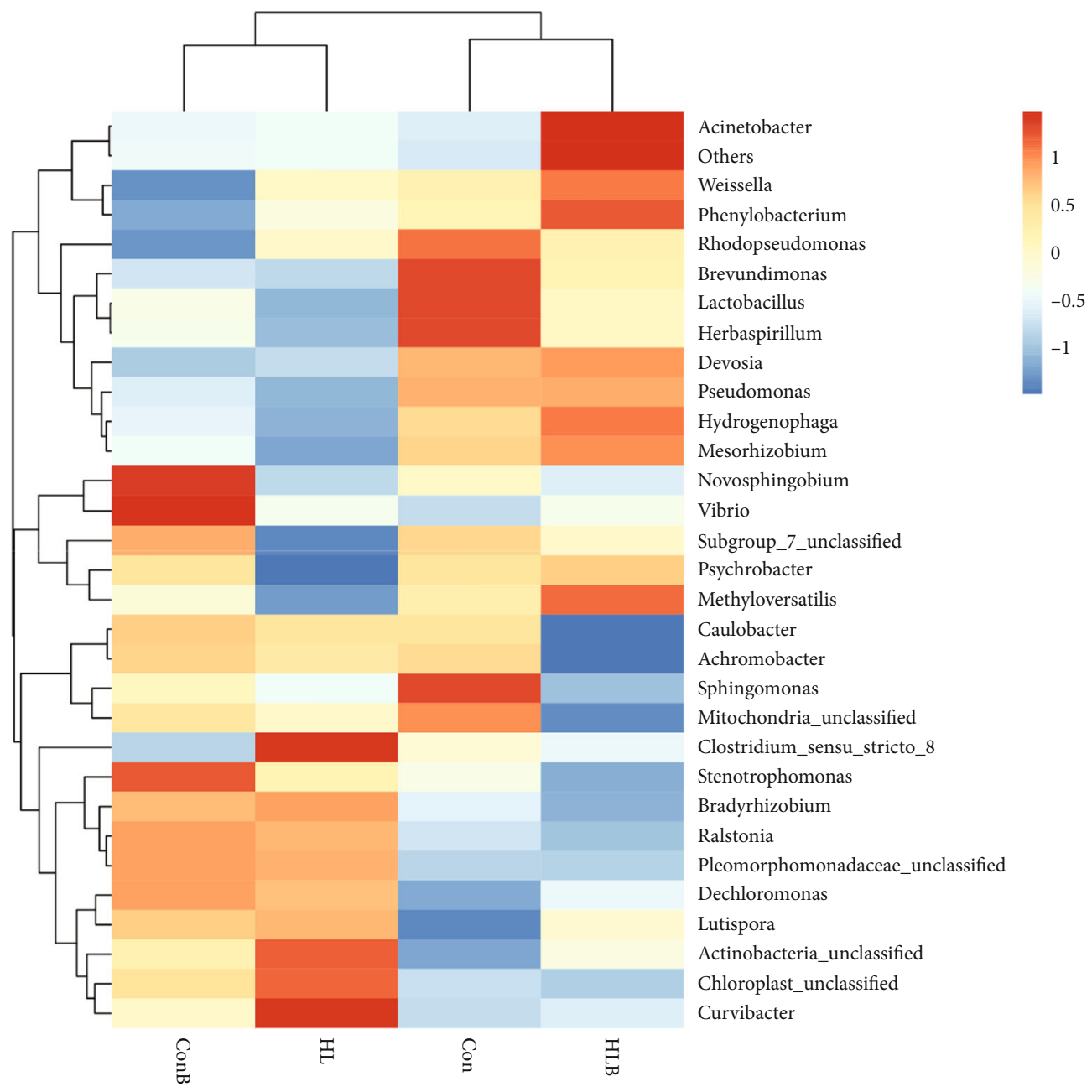

Figure 7: Heatmap of the abundance at genus level of black sea bream fed different experimental diets $(n=3)$. Color and color intensity reveal the relatively enrichment of OUT. Con: control group; ConB: control diet with $50 \mathrm{mg} / \mathrm{kg}$ berberine treatment; HL: high-lipid group; HLB: high-lipid diet with $50 \mathrm{mg} / \mathrm{kg}$ berberine treatment.

phylum Bacteroidetes abundance was increased by a high-fat diet in zebrafish [24]. However, grass carp fed a berberinesupplemented diet for 28 days had a relatively increased abundance of Firmicutes, Bacteroidetes, and Proteobacteria in the intestine [3]. A study in hypercholesteraemic hamsters found that berberine treatment could increase the Firmicutes-to-Bacteroidete $(\mathrm{F} / \mathrm{B})$ ratio [53], which is associated with intestinal energy absorption, and a higher ratio could be an indication of more intestinal energy harvest $[15,54]$. In the present study, berberine supplementation in the normal diet decreased intestinal $\mathrm{F} / \mathrm{B}$ ratio but increased it when berberine was supplemented in the highlipid diet. Decreased intestinal or fecal F/B ratio by berberine treatment was reported in high-fat diet-fed rats [55] and mice [16]. However, berberine treatment improved the intestinal $\mathrm{F} / \mathrm{B}$ ratio in diabetes rats [56]. The variances of the $\mathrm{F} / \mathrm{B}$ ratio by berberine treatment deserve further study.

In the present study, berberine supplementation in the high-lipid diet restored intestinal Pseudomonas richness decreased by high-lipid diet (Table 7). A study in Pacific white shrimp (Litopenaeus vannamei) found that supplementation of probiotic Bacillus coagulans increased Pseudo- monas community in the intestine [57]. Besides, berberine was found to inhibit some opportunistic pathogens' growth, such as Prevotella and Proteus, but increased the abundance of some probiotics, like Lactobacillus and Akkermansia [58]. In this study, Lactobacillus and Weissella abundances in the HLB and Con groups were higher than that of the HL group. These two bacteria are probiotic candidates belonging to lactic acid bacteria and can retard intestinal pathogenic colonization in the epithelial layer and enhance fish resistance to infection [59]. Increased Lactobacillus and Weissella abundance was reported in turbot-fed fructooligosaccharidesupplemented diet [59]. Besides, administration of Lactobacillus improved growth performance has been reported in Nile tilapia (Oreochromis niloticus) [60], grass carp [61], and black sea bream [62]. And replacement of fishmeal with meat and bone meal reduced intestinal Weissella abundance of gilthead sea bream (Sparus aurata) [63]. According to the LEfSe result, Actinobacteria abundance was higher in the HL group than that of the HLB group; a similar result was also found in obese rats treated with berberine [13]. In addition, the abundances of Ruegeria, Devosia, and Rhodobacteraceae were higher in the HLB group than that of the other groups. 

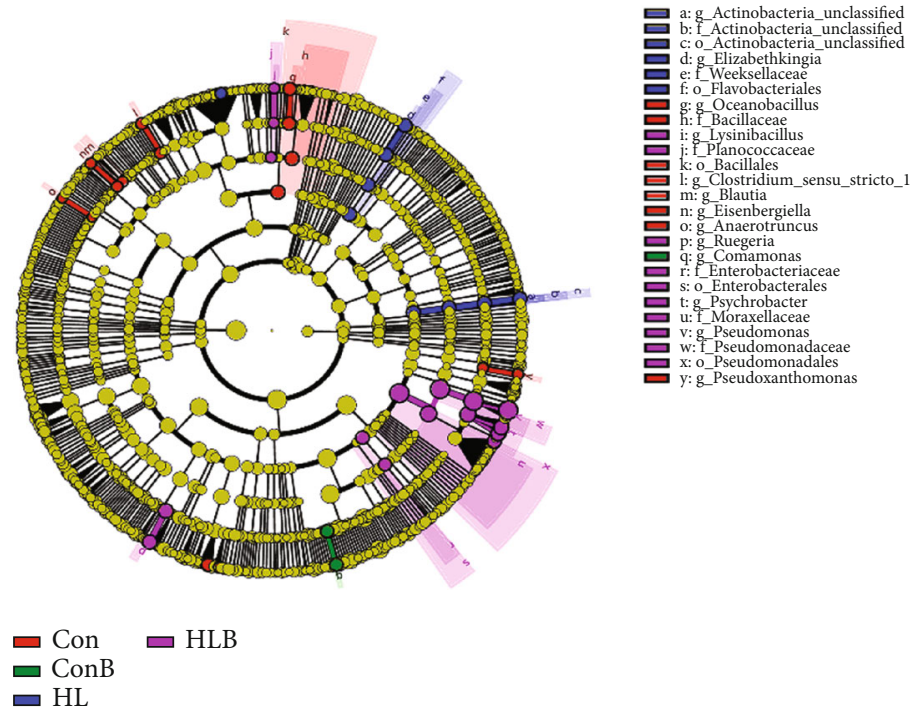

(a)

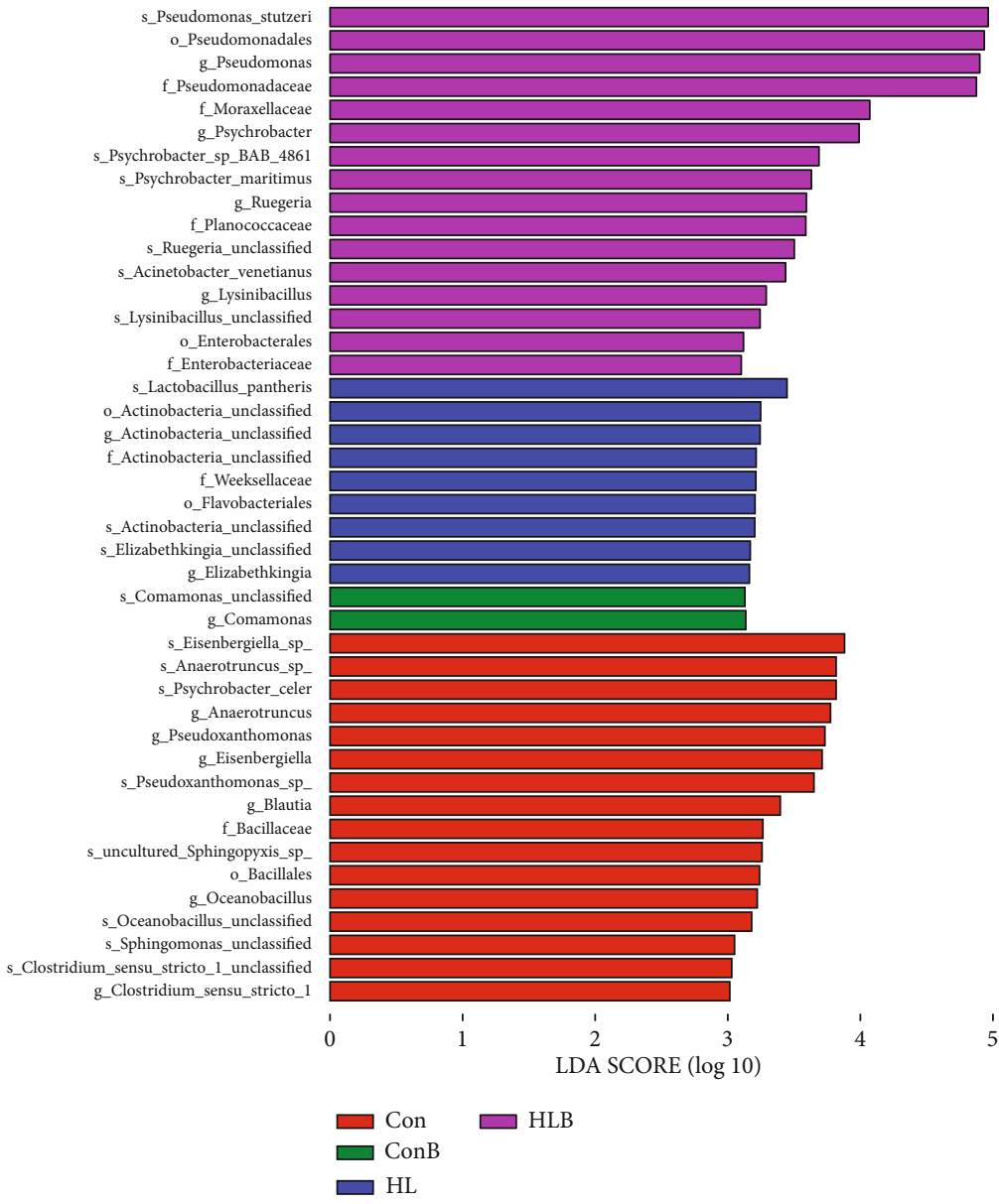

(b)

FIGURE 8: LEfSe analysis of intestinal microbiota communities of black sea bream fed different diets. LEfSe taxonomic cladogram (a). The diameter of each circle was based on the taxon's abundance. Cladogram was calculated by LefSe and displayed according to effect size. Node size is proportional to the average abundance; colour indicates the relative concentration of the clusters. Con: control group; ConB: control diet with $50 \mathrm{mg} / \mathrm{kg}$ berberine treatment; HL: high-lipid group; HLB: high-lipid diet with $50 \mathrm{mg} / \mathrm{kg}$ berberine treatment. Histogram of linear discriminant analysis (LDA) scores for differentially abundant taxon (b). Different colors suggest the highest enrichment of certain taxa in Con (red), ConB (green), HL (blue), and HLB (purple). 
Ruegeria is a Gram-negative bacteria and belongs to the class Alphaproteobacteria [64]. It is used as a probiotic and symbiotic with unicellular eukaryotic phytoplankton [65]. Dietary tributyrin supplementation in a high soya bean meal diet increased intestinal Devosia abundance in yellow drum [49]. Rhodobacteraceae belongs to photosynthetic bacteria that contain abundant functional factors and nutrients, indicating it could be a potential probiotic $[66,67]$. However, it is unclear why berberine supplementation in the normal diet modulated intestinal microbiota composition similar to that of the HL group, which deserves further study.

\section{Conclusion}

In conclusion, berberine supplementation in high-lipid diet downregulated liver proinflammatory cytokine expressions but promoted anti-inflammatory cytokine expressions, improved intestinal villus length and muscularis mucosae thickness, rebalanced intestinal microbiota diversity, and promoted some intestinal probiotic growth of black sea bream. $50 \mathrm{mg} / \mathrm{kg}$ dietary berberine was a safe and effective dosage for alleviating high dietary lipid stress in the liver and intestine. However, the alleviation effect of berberine may vary with dietary lipid and berberine levels, which deserves further study.

\section{Data Availability}

Derived data supporting this study are available from the corresponding author on reasonable request.

\section{Conflicts of Interest}

The authors declare that they have no conflicts of interest.

\section{Authors' Contributions}

Lei Wang and Qingjun Shao designed this experiment. Gladstone Sagada, Bingying Xu, and Jinzhi Zhang provided help during the feeding trial and sampling. Lei Wang wrote this manuscript, and Gladstone Sagada helped revise it. All authors read and approved the final manuscript.

\section{Acknowledgments}

This work was funded by the Ministry of Science and Technology, China (Project No: 2020YFD0900801) and the Natural Science Foundation of Universities of Anhui Province (Project No: KJ2021A0105).

\section{References}

[1] F. Zuo, N. Nakamura, T. Akao, and M. Hattori, "Pharmacokinetics of berberine and its main metabolites in conventional and pseudo germ-free rats determined by liquid chromatography/ion trap mass spectrometry," Drug Metabolism and Disposition, vol. 34, no. 12, pp. 2064-2072, 2006.

[2] Y. Wang, J. W. Shou, X. Y. Li et al., "Berberine-induced bioactive metabolites of the gut microbiota improve energy metab- olism," Metabolism, Clinical and Experimental, vol. 70, pp. 72-84, 2017.

[3] H. Pan, Z. Li, J. Xie et al., "Berberine influences blood glucose via modulating the gut microbiome in Grass carp," Frontiers in Microbiology, vol. 10, no. 1066, 2019.

[4] C. Chen, Z. Yu, Y. Li, J. Fichna, and M. Storr, "Effects of berberine in the gastrointestinal tract - a review of actions and therapeutic implications," The American Journal of Chinese Medicine, vol. 42, no. 5, pp. 1053-1070, 2014.

[5] J.-M. Hur, M.-S. Hyun, S.-Y. Lim, W.-Y. Lee, and D. Kim, "The combination of berberine and irradiation enhances anticancer effects via activation of p38 MAPK pathway and ROS generation in human hepatoma cells," Journal of Cellular Biochemistry, vol. 107, no. 5, pp. 955-964, 2009.

[6] W.-J. Shan, L. Huang, Q. Zhou, F.-C. Meng, and X.-S. Li, "Synthesis, biological evaluation of $9-\mathrm{N}$-substituted berberine derivatives as multi-functional agents of antioxidant, inhibitors of acetylcholinesterase, butyrylcholinesterase and amyloid- $\beta$ aggregation," European Journal of Medicinal Chemistry, vol. 46, no. 12, pp. 5885-5893, 2011.

[7] Y. L. Siow, L. Sarna, and K. O, "Redox regulation in health and disease - Therapeutic potential of berberine," Food Research International, vol. 44, no. 8, pp. 2409-2417, 2011.

[8] B. Pang, L.-H. Zhao, Q. Zhou et al., "Application of berberine on treating type 2 diabetes mellitus," International Journal of Endocrinology, vol. 2015, Article ID 905749, 12 pages, 2015.

[9] Y. Suo, E. Li, T. Li et al., "Response of gut health and microbiota to sulfide exposure in Pacific white shrimp Litopenaeus vannamei," Fish \& Shellfish Immunology, vol. 63, pp. 87-96, 2017.

[10] P. Sun, M. Jin, L. Ding et al., "Dietary lipid levels could improve growth and intestinal microbiota of juvenile swimming crab, Portunus trituberculatus," Aquaculture, vol. 490, pp. 208-216, 2018.

[11] X. Zheng, Y. Duan, H. Dong, and J. Zhang, "The effect of Lactobacillus plantarum administration on the intestinal microbiota of whiteleg shrimp Penaeus vannamei," Aquaculture, vol. 526, p. 735331, 2020.

[12] T. Li, M. Long, C. Ji et al., "Alterations of the gut microbiome of largemouth bronze gudgeon (Coreius guichenoti) suffering from furunculosis," Scientific Reports, vol. 6, no. 1, p. 30606, 2016.

[13] X. Zhang, Y. Zhao, M. Zhang et al., "Structural changes of gut microbiota during berberine-mediated prevention of obesity and insulin resistance in high-fat diet-fed rats," PLoS One, vol. 7, no. 8, article e42529, 2012.

[14] R. Sun, N. Yang, B. Kong et al., "Orally administered berberine modulates hepatic lipid metabolism by altering microbial bile acid metabolism and the intestinal FXR signaling pathway," Molecular Pharmacology, vol. 91, no. 2, pp. 110-122, 2017.

[15] R. Jumpertz, D. S. Le, P. J. Turnbaugh et al., "Energy-balance studies reveal associations between gut microbes, caloric load, and nutrient absorption in humans," The American Journal of Clinical Nutrition, vol. 94, no. 1, pp. 58-65, 2011.

[16] W. Xie, D. Gu, J. Li, K. Cui, and Y. Zhang, "Effects and action mechanisms of berberine and Rhizoma coptidis on gut microbes and obesity in high-fat diet-fed C57BL/6J mice," PLoS One, vol. 6, no. 9, pp. e24520-e24520, 2011.

[17] C. Yu, J. Zhang, Q. Qin, J. Liu, J. Xu, and W. Xu, "Berberine improved intestinal barrier function by modulating the intestinal microbiota in blunt snout bream (Megalobrama 
amblycephala) under dietary high-fat and high-carbohydrate stress," Fish \& Shellfish Immunology, vol. 102, pp. 336-349, 2020.

[18] B. S. Kamalam, F. Medale, and S. Panserat, "Utilisation of dietary carbohydrates in farmed fishes: new insights on influencing factors, biological limitations and future strategies," Aquaculture, vol. 467, pp. 3-27, 2017.

[19] L. Wang, B. Xu, G. Sagada et al., "Dietary berberine regulates lipid metabolism in muscle and liver of black sea bream (Acanthopagrus schlegelii) fed normal or high-lipid diets," British Journal of Nutrition, vol. 125, no. 5, pp. 481-493, 2021.

[20] D. R. Tocher, "Metabolism and functions of lipids and fatty acids in teleost fish," Reviews in Fisheries Science, vol. 11, no. 2, pp. 107-184, 2003.

[21] L. Wang, W. Zhang, S. Gladstone, W.-K. Ng, J. Zhang, and Q. Shao, "Effects of isoenergetic diets with varying protein and lipid levels on the growth, feed utilization, metabolic enzymes activities, antioxidative status and serum biochemical parameters of black sea bream (Acanthopagrus schlegelii)," Aquaculture, vol. 513, p. 734397, 2019.

[22] K.-L. Lu, L.-N. Wang, D.-D. Zhang, W.-B. Liu, and W.-N. Xu, "Berberine attenuates oxidative stress and hepatocytes apoptosis via protecting mitochondria in blunt snout bream Megalobrama amblycephala fed high-fat diets," Fish Physiology and Biochemistry, vol. 43, no. 1, pp. 65-76, 2017.

[23] M. Peng, J. Xue, Y. Hu et al., "Disturbance in the homeostasis of intestinal microbiota by a high-fat diet in the rice field eel (Monopterus albus)," Aquaculture, vol. 502, pp. 347-355, 2019.

[24] N. Arias-Jayo, L. Abecia, L. Alonso-Sáez, A. Ramirez-Garcia, A. Rodriguez, and M. A. Pardo, "High-fat diet consumption induces microbiota dysbiosis and intestinal inflammation in zebrafish," Microbial Ecology, vol. 76, no. 4, pp. 1089-1101, 2018.

[25] Q.-Q. Chen, W.-B. Liu, M. Zhou et al., "Effects of berberine on the growth and immune performance in response to ammonia stress and high-fat dietary in blunt snout bream Megalobrama amblycephala," Fish \& Shellfish Immunology, vol. 55, pp. 165172, 2016.

[26] W. Zhou, S. Rahimnejad, K. Lu, L. Wang, and W. Liu, "Effects of berberine on growth, liver histology, and expression of lipidrelated genes in blunt snout bream (Megalobrama amblycephala) fed high-fat diets," Fish Physiology and Biochemistry, vol. 45, no. 1, pp. 83-91, 2019.

[27] B. Chen, Y.-M. Zheng, M.-Q. Zhang, Y. Han, J.-P. Zhang, and C.-Q. Hu, "Microarray expression profiling and raman spectroscopy reveal anti-fatty liver action of berberine in a dietinduced larval zebrafish model," Frontiers in Pharmacology, vol. 10, no. 1504, p. 1504, 2020.

[28] K. J. Livak and T. D. Schmittgen, "Analysis of relative gene expression data using real-time quantitative PCR and the $2^{-\Delta \Delta \mathrm{CT}}$ method," Methods, vol. 25, no. 4, pp. 402-408, 2001.

[29] M. Jin, T. Pan, X. Cheng et al., "Effects of supplemental dietary l-carnitine and bile acids on growth performance, antioxidant and immune ability, histopathological changes and inflammatory response in juvenile black seabream (Acanthopagrus schlegelii) fed high-fat diet," Aquaculture, vol. 504, pp. 199209, 2019.

[30] M. Jin, T. Pan, D. R. Tocher et al., "Dietary choline supplementation attenuated high-fat diet-induced inflammation through regulation of lipid metabolism and suppression of $\mathrm{NF} \kappa \mathrm{B}$ activation in juvenile black seabream (Acanthopagrus schlegelii)," Journal of Nutritional Science, vol. 8, article e38, 2019.
[31] M. Jin, T. Zhu, D. R. Tocher et al., "Dietary fenofibrate attenuated high-fat-diet-induced lipid accumulation and inflammation response partly through regulation of _ppar $\alpha_{-}$and _sirt1_in juvenile black seabream (Acanthopagrus schlegelii)," Developmental \& Comparative Immunology, vol. 109, p. 103691, 2020.

[32] P. Tan, M. Peng, D. Liu et al., "Suppressor of cytokine signaling 3 (SOCS3) is related to pro-inflammatory cytokine production and triglyceride deposition in turbot (Scophthalmus maximus)," Fish \& Shellfish Immunology, vol. 70, pp. 381-390, 2017.

[33] J. Yang, X. J. Ma, L. Li et al., "Berberine ameliorates nonalcoholic steatohepatitis in ApoE $<$ sup $>-/-</$ sup $>$ mice," Experimental and Therapeutic Medicine, vol. 14, no. 5, pp. 4134-4140, 2017.

[34] J. Zhao, Y. Wang, X. Wu et al., "Inhibition of CCL19 benefits non-alcoholic fatty liver disease by inhibiting TLR4/NF- $\kappa \mathrm{B}-$ p65 signaling," Molecular Medicine Reports, vol. 18, no. 5, pp. 4635-4642, 2018.

[35] B.-H. Choi, I. S. Ahn, Y.-H. Kim et al., "Berberine reduces the expression of adipogenic enzymes and inflammatory molecules of 3T3-L1 adipocyte," Experimental \& Molecular Medicine, vol. 38, pp. 599-605, 2006.

[36] J. H. Xu, X. Z. Liu, W. Pan, and D. J. Zou, "Berberine protects against diet-induced obesity through regulating metabolic endotoxemia and gut hormone levels," Molecular Medicine Reports, vol. 15, no. 5, pp. 2765-2787, 2017.

[37] L. Wang, Y. Sun, B. Xu et al., "Effects of berberine supplementation in high starch diet on growth performance, antioxidative status, immune parameters and ammonia stress response of fingerling black sea bream (Acanthopagrus schlegelii)," Aquaculture, vol. 527, p. 735473, 2020.

[38] Y. Wang, J. Zheng, H. Hou et al., "Effects of berberine on intestinal flora of non-alcoholic fatty liver induced by high-fat diet through 16S rRNA gene segmentation," Journal of King Saud University-Science, vol. 32, no. 5, pp. 2603-2609, 2020.

[39] W.-N. Xu, D.-H. Chen, Q.-Q. Chen, and W.-B. Liu, "Growth performance, innate immune responses and disease resistance of fingerling blunt snout bream, Megalobrama amblycephala adapted to different berberine-dietary feeding modes," Fish \& Shellfish Immunology, vol. 68, pp. 458-465, 2017.

[40] S.-M. Lin, X.-M. Zhou, Y.-L. Zhou et al., "Intestinal morphology, immunity and microbiota response to dietary fibers in largemouth bass, Micropterus salmoide," Fish \& Shellfish Immunology, vol. 103, pp. 135-142, 2020.

[41] C. Erridge, T. Attina, C. M. Spickett, and D. J. Webb, "A highfat meal induces low-grade endotoxemia: evidence of a novel mechanism of postprandial inflammation," The American Journal of Clinical Nutrition, vol. 86, no. 5, pp. 1286-1292, 2007.

[42] C.-S. Liu, Y.-R. Zheng, Y.-F. Zhang, and X.-Y. Long, "Research progress on berberine with a special focus on its oral bioavailability," Fitoterapia, vol. 109, pp. 274-282, 2016.

[43] B. Deplancke and H. R. Gaskins, "Microbial modulation of innate defense: goblet cells and the intestinal mucus layer," American Journal of Clinical Nutrition, vol. 73, no. 6, pp. 1131S-1141S, 2001.

[44] W.-G. Kong, S.-S. Li, X.-X. Chen, Y.-Q. Huang, Y. Tang, and Z.-X. Wu, "A study of the damage of the intestinal mucosa barrier structure and function of Ctenopharyngodon idella with Aeromonas hydrophila," Fish Physiology and Biochemistry, vol. 43, no. 5, pp. 1223-1235, 2017. 
[45] P. A. UrÁN, J. W. Schrama, J. H. W. M. Rombout et al., "Soybean meal-induced enteritis in Atlantic salmon (Salmo salar L.) at different temperatures," Aquaculture Nutrition, vol. 14, no. 4, pp. 324-330, 2008.

[46] M. Schoeler and R. Caesar, "Dietary lipids, gut microbiota and lipid metabolism," Reviews in Endocrine \& Metabolic Disorders, vol. 20, no. 4, pp. 461-472, 2019.

[47] F. Vargas-Albores, L. R. Martínez-Córdova, A. HernándezMendoza, F. Cicala, A. Lago-Lestón, and M. MartínezPorchas, "Therapeutic modulation of fish gut microbiota, a feasible strategy for aquaculture?," Aquaculture, vol. 544, p. $737050,2021$.

[48] E. le Chatelier, T. Nielsen, J. Qin et al., "Richness of human gut microbiome correlates with metabolic markers," Nature, vol. 500, no. 7464, pp. 541-546, 2013.

[49] P. Tan, X. Wu, W. Zhu, B. Lou, R. Chen, and L. Wang, "Effect of tributyrin supplementation in high-soya bean meal diet on growth performance, body composition, intestine morphology and microbiota of juvenile yellow drum (Nibea albiflora)," Aquaculture Research, vol. 51, no. 5, pp. 2004-2019, 2020.

[50] C. Zhang, X. Zheng, X. Ren, Y. Li, and Y. Wang, "Bacterial diversity in gut of large yellow croaker Larimichthys crocea and black sea bream Sparus macrocephalus reared in an inshore net pen," Fisheries Science, vol. 85, no. 6, pp. 10271036, 2019.

[51] M. Zhang, Y. Sun, L. Chen et al., "Symbiotic bacteria in gills and guts of Chinese mitten crab (Eriocheir sinensis) differ from the free-living bacteria in water," PLoS One, vol. 11, no. 1, article e0148135, 2016.

[52] H. Abdelhamed, O. Ozdemir, G. Waldbieser, A. D. Perkins, M. L. Lawrence, and A. Karsi, "Effects of florfenicol feeding on diversity and composition of the intestinal microbiota of channel catfish (Ictalurus punctatus)," Aquaculture Research, vol. 50, no. 12, pp. 3663-3672, 2019.

[53] S. Gu, B. Cao, R. Sun et al., "A metabolomic and pharmacokinetic study on the mechanism underlying the lipid-lowering effect of orally administered berberine," Molecular BioSystems, vol. 11, no. 2, pp. 463-474, 2015.

[54] Y. Cao, Q. Pan, W. Cai et al., "Modulation of gut microbiota by berberine improves steatohepatitis in high-fat diet-fed BALB/ C mice," Archives of Iranian Medicine, vol. 19, no. 3, pp. 197-203, 2016.

[55] H. Sun, N. Wang, Z. Cang et al., "Modulation of microbiotagut-brain axis by berberine resulting in improved metabolic status in high-fat diet-fed rats," Obesity Facts, vol. 9, no. 6, pp. 365-378, 2016.

[56] X. Xu, Z. Gao, F. Yang et al., "Antidiabetic effects of gegen qinlian decoction via the gut microbiota are attributable to its key ingredient berberine," Genomics, Proteomics \& Bioinformatics, vol. 18, no. 6, pp. 721-736, 2020.

[57] K. Amoah, Q.-C. Huang, B.-P. Tan et al., "Dietary supplementation of probiotic_Bacillus coagulans_ATCC 7050, improves the growth performance, intestinal morphology, microflora, immune response, and disease confrontation of Pacific white shrimp, _Litopenaeus vannamei," Fish \& Shellfish Immunology, vol. 87, pp. 796-808, 2019.

[58] W. Zhang, J.-H. Xu, T. Yu, and Q.-K. Chen, "Effects of berberine and metformin on intestinal inflammation and gut microbiome composition in $\mathrm{db} / \mathrm{db}$ mice," Biomedicine and Pharmacotherapy, vol. 118, p. 109131, 2019.
[59] I. Guerreiro, P. Enes, A. Rodiles, D. Merrifield, and A. OlivaTeles, "Effects of rearing temperature and dietary short-chain fructooligosaccharides supplementation on allochthonous gut microbiota, digestive enzymes activities and intestine health of turbot (Scophthalmus maximus L.) juveniles," Aquaculture Nutrition, vol. 22, no. 3, pp. 631-642, 2016.

[60] N. Van Nguyen, S. Onoda, T. Van Khanh et al., "Evaluation of dietary heat-killed Lactobacillus plantarum strain L-137 supplementation on growth performance, immunity and stress resistance of Nile tilapia (Oreochromis niloticus)," Aquaculture, vol. 498, pp. 371-379, 2019.

[61] G. Guo, C. Li, B. Xia et al., "The efficacy of lactic acid bacteria usage in turbot Scophthalmus maximus on intestinal microbiota and expression of the immune related genes," Fish \& Shellfish Immunology, vol. 100, pp. 90-97, 2020.

[62] G. Sagada, N. Gray, L. Wang et al., "Effect of dietary inactivated_Lactobacillus plantarum_ on growth performance, antioxidative capacity, and intestinal integrity of black sea bream (Acanthopagrus schlegelii) fingerlings.," Aquaculture, vol. 535, p. 736370, 2021.

[63] S. Moutinho, H. Peres, C. Serra et al., "Meat and bone meal as partial replacement of fishmeal in diets for gilthead sea bream (Sparus aurata) juveniles: diets digestibility, digestive function, and microbiota modulation," Aquaculture, vol. 479, pp. 721731, 2017.

[64] L. Fan and Q. X. Li, "Characteristics of intestinal microbiota in the Pacific white shrimp Litopenaeus vannamei differing growth performances in the marine cultured environment," Aquaculture, vol. 505, pp. 450-461, 2019.

[65] F. Barreto-Curiel, S. T. Ramirez-Puebla, E. Ring $\varnothing$ et al., "Effects of extruded aquafeed on growth performance and gut microbiome of juvenile_Totoaba macdonaldi," Animal Feed Science and Technology, vol. 245, pp. 91-103, 2018.

[66] M. Zhang, Y. Sun, K. Chen et al., "Characterization of the intestinal microbiota in Pacific white shrimp, Litopenaeus vannamei, fed diets with different lipid sources," Aquaculture, vol. 434, pp. 449-455, 2014.

[67] X. X. Zhou, Y. J. Pan, Y. B. Wang, and W. F. Li, "In vitro assessment of gastrointestinal viability of two photosynthetic bacteria, Rhodopseudomonas palustris and Rhodobacter sphaeroides," Journal of Zhejiang University. Science. B, vol. 8, no. 9, pp. 686-692, 2007. 